\title{
GENERIC REALIGNMENTS IN MAXILLARIINAE (ORCHIDACEAE)
}

\author{
Mario A. Blanco ${ }^{1,2}$, German Carnevali ${ }^{3}$, W. Mark Whitten ${ }^{4}$, Rodrigo B. Singer ${ }^{5}$, \\ Samantha KoehleR ${ }^{6}$, Norris H. Williams ${ }^{4}$, Isidro OJedA ${ }^{7}$, Kurt M. NeUbig ${ }^{1}$ \\ \& LORENA ENDARA ${ }^{1}$
}

${ }^{1}$ Department of Botany, University of Florida, 220 Bartram Hall, Gainesville, FL 32611-8526, USA. (mablanco@ufl.edu,kneubig@ufl.edu, lendara@flmnh.ufl.edu)

²Jardín Botánico Lankester, Universidad de Costa Rica, Apdo. 1031-7050, Cartago, Costa Rica

${ }^{3}$ Herbario CICY, Centro de Investigación Científica de Yucatán (CICY), calle 43 No. 130,

Col. Chuburná de Hidalgo, 97200 Mérida, Yucatán, México. (carneval@cicy.mx)

${ }^{4}$ Florida Museum of Natural History, University of Florida, P.O. Box 117800, Gainesville, FL 32611-7800, USA

(whitten@flmnh.ufl.edu, orchid@flmnh.ufl.edu)

${ }^{5}$ Departamento de Botânica, Instituto de Biociências, Universidade Federal de Rio Grande do Sul, CEP 91501-970, Porto Alegre, RS, Brazil. (rbsinger1@yahoo.com)

${ }^{6}$ Departamento de Genética, Escola Superior de Agricultura "Luiz de Queiróz", Universidade de São Paulo,

C.P. 83, Piracicaba, SP, Brazil 13400-970. (samantha.koehler@gmail.com)

${ }^{7}$ Center for Plant Research, University of British Columbia, \#302-Macmillan Building, 2357 Main Mall, Vancouver, B.C., Canada V6T 1Z4. (isidro@interchange.ubc.ca)

ABSTRACT. A recent phylogenetic analysis of four DNA regions for ca. 354 species of core Maxillariinae strongly indicate that the genus Maxillaria, as traditionally circumscribed, is grossly polyphyletic. We present a new phylogenetic classification for core Maxillariinae that recognizes 17 genera. Necessary realignments include: 1) resurrection of the genera Camaridium, Heterotaxis, and Ornithidium; 2) recognition of the recent segregates Brasiliorchis (=Maxillaria sect. Repentes), Christensonella (=Maxillaria sect. Urceolatae), Nitidobulbon (in press), and a recircumscribed Sauvetrea (=Maxillaria sect. Trigonae); 3) adoption of the new genera Inti (=Maxillaria sect. Polyphyllae), Mapinguari, Maxillariella (=Maxillaria sections Ebulbes and Erectae), and Rhetinantha; 4) transfers from Maxillaria sect. Reflexae to Ornithidium, and Maxillaria sect. Rufescens to Mormolyca; and 5) synonymizing of the genera Adamanthus, Pseudomaxillaria, Psittacoglossum, and Sepalosaccus (under Camaridium), Anthosiphon (under Cryptocentrum), Chrysocycnis (under Mormolyca), Dicrypta, Marsupiaria, and Pentulops (under Heterotaxis), and Laricorchis, Neo-urbania, and Siagonanthus (under Ornithidium). Some new synonyms at the specific level are also presented.

RESUMEN. Un reciente análisis filogenético de cuatro regiones de ADN para ca. 354 especies de la subtribu Maxillariinae indican fuertemente que el género Maxillaria, en su circunscripción tradicional, es altamente polifilético. Presentamos una nueva clasificación filogenética para Maxillariinae que reconoce 17 géneros. Los cambios necesarios incluyen: 1) la resurrección de los géneros Camaridium, Heterotaxis, y Ornithidium; 2) el reconocimiento de los recientes segregados genéricos Brasiliorchis (=Maxillaria sección Repentes), Christensonella (=Maxillaria sección Urceolatae), Nitidobulbon (en prensa), y una Sauvetrea recircunscrita (=Maxillaria sección Trigonae); 3) la adopción de los nuevos géneros Inti (=Maxillaria sección Polyphyllae), Mapinguari, Maxillariella (=Maxillaria secciones Ebulbes y Erectae), y Rhetinantha; 4) transferencias de Maxillaria sección Reflexae a Ornithidium, y Maxillaria sección Rufescens a Mormolyca; y 5) puesta en sinonimia de los géneros Adamanthus, Pseudomaxillaria, Psittacoglossum y Sepalosaccus (bajo Camaridium), Anthosiphon (bajo Cryptocentrum), Chrysocycnis (bajo Mormolyca), Dicrypta, Marsupiaria y Pentulops (bajo Heterotaxis), y Laricorchis, Neo-Urbania, y Siagonanthus (bajo Ornithidium). Algunos sinónimos nuevos al nivel de especie también son presentados.

Key words: Cymbidieae, generic recircumscription, Inti, Mapinguari, Maxillariella, Maxillariinae, Orchidaceae, Rhetinantha. 
Subtribe Maxillariinae (sensu Chase et al. 2003) includes a number of genera endemic to the Neotropics. The circumscription of genera in the core Maxillariinae (sensu Whitten et al. 2007, equal to Maxillariinae sensu Dressler, 1993) has been problematic since the creation of the genus Maxillaria by Ruiz and Pavón $(1794,1798)$. This large genus (ca. 580 species as traditionally defined; e.g., Govaerts et al. 2005) has for a long time been considered an assemblage of morphologically disparate taxa (Christenson 2002a, 2002b; Whitten et al. 2007 and references therein), and thus probably non-monophyletic. The complicated species-level taxonomy of Maxillaria has hampered attempts of providing a subgeneric classification (Christenson 2002a, 2002b $\mathrm{b}^{1}$ ), a situation mirrored in other large plant genera (Pfeil \& Crisp 2005, Monro 2006, Parnell et al. 2007).

The recent molecular phylogenetic analysis of Dathe \& Dietrich (2006) provided the first concrete evidence for the grossly polyphyletic nature of Maxillaria. However, the limited taxon sampling (30 species, one individual each) and low number of DNA regions used in their study (nrITS only) precluded any sound taxonomic decisions based on their results. More recently, Whitten et al. (2007) presented a vastly more detailed phylogenetic analysis of core Maxillariinae (619 individuals representing ca. 354 species; four DNA regions used) that corroborated Dathe and Dietrich's preliminary results and revealed a worst-case scenario: all the currently accepted minor genera of core Maxillariinae

${ }^{1}$ Christenson (2002a) presented a partial subgeneric classification of Maxillaria with 19 sections, 13 of them newly proposed. A French translation of the same article (Christenson 2002b) was meant to appear much later, but the publication of the Proceedings of the $16^{\text {th }}$ World Orchid Conference (here abbreviated "Proc. 16 ${ }^{\text {th }}$ World Orchid Conf.") was delayed substantially. The two publications appeared in 2006, and the exact date of printing is not indicated in either one. The Proceedings were published ca. six weeks before the translation in Richardiana, and thus constitute the place of valid publication of most of Christenson's sections. The only exception is section Ornithidium, which missed the basionym citation in the Proceedings, and was validated in Richardiana (E. A. Christenson, personal comunication 2007).
(Anthosiphon, Cryptocentrum, Chrysocycnis, Cyrtidiorchis, Mormolyca, Pityphyllum, and Trigonidium, Govaerts et al. 2005), are firmly nested in Maxillaria sensu lato. Thus, the current generic classification in core Maxillariinae is untenable on phylogenetic grounds.

Backlund \& Bremer (1998) presented guidelines on how to modify existing classifications in order to comply with phylogenetic requirements. Their primary principle is to designate only monophyletic groups as ranked taxa. Secondary principles (in no particular order of importance) are: a) to designate only highly supported clades as ranked taxa; b) to minimize nomenclatural disruption; c) to designate easily recognizable clades as ranked taxa; and d) to minimize taxonomic redundancy by lumping monotypic taxa with their sister group, if feasible. Entwisle \& Weston (2005) independently advanced similar guidelines, although they also recommended to minimize taxonomic change in "charismatic" or economically and/or horticulturally important groups, and to avoid the use of epithets already in existence in potential congeners (when creating new names or describing new species). Entwisle \& Weston (2005) concluded that ease of recognition of genera is not always achievable, and that certain "biological criteria" (e.g., hybridization, special morphological characters) should not be emphasized when designating genera. In the new classification presented here, we attempt to follow these guidelines.

We favor the recognition of 17 genera in core Maxillariinae (clades A through Q in Whitten et al. 2007) that correspond to well supported clades (fig. 1). This inevitably requires the resurrection of some generic names previously placed in synonymy with Maxillaria, and the creation of several new genera. These new generic realignments are presented here. The alternative, to lump the minor genera in Maxillaria, would be less nomenclaturally disruptive, but would substantially add to the already baffling morphological diversity of that genus and make it even less morphologically diagnosable. Further arguments in support of our new classification and morphological characters for each genus are presented in Whitten et al. (2007). Keys and detailed descriptions to all the genera in subtribe Maxillariinae will be published elsewhere. 


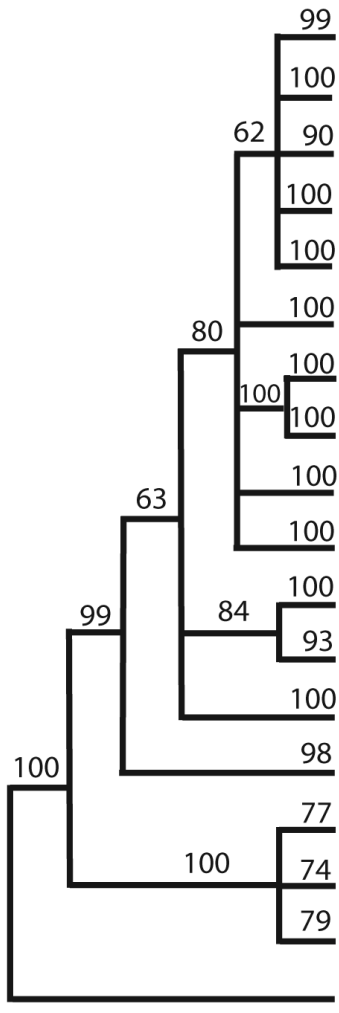

Maxillaria $200-250$ Camaridium 80 Trigonidium 7 Maxillariella 50 Rhetinantha 15 Mormolyca 25 Cyrtidiorchis 5 Sauvetrea 15 Christensonella 12 Mapinguari 4 Cryptocentrum 18 Inti 2 Brasiliorchis 13 Pityphyllum 7 Ornithidium 60 Heterotaxis 13 Nitidobulbon 3

\section{Outgroups}

FIGURE 1. Summary of generic relationships within the core Maxillariinae, simplified from figure 8 of Whitten et al. (2007). Based on a maximum parsimony analysis of combined nrITS, mat $K+\operatorname{trn} K$, atpB-rbcL spacer, and rpoC1 DNA sequence data. Values above branches are bootstrap percentages. Numbers next to generic names indicate their known or estimated number of species.

The realization that many large and/or charismatic genera are polyphyletic or need to be lumped with others based on phylogenetic principles has recently spurred support from part of the botanical community for the acceptance of paraphyletic taxa (most of the debate has taken place in recent issues of Taxon). However, we are convinced that the designation of monophyletic genera is a far better, less subjective option. Discussion of our arguments for this lies outside of the realm of the present contribution; we refer readers to Pfeil \& Crisp (2005), who provide a lucid argument in favor of phylogenetic classifications and against the use of paraphyletic supraspecific taxa.

We also effect the nomenclatural transfers required by our new classification. Following Cribb et al.'s (1985) recommendation, we only transfer species for which we are confident of their systematic position either because we have sampled them for our molecular phylogeny (Whitten et al. 2007) or because of their clear morphological affinity with species included in our analyses.

We refrain from transferring names that might be synonyms (even if not yet currently regarded as such), and treat taxonomically complicated groups with caution. We do not transfer a few names that appear in our phylogenies (Whitten et al. 2007) because now we consider them as synonyms. Many species of Camaridium and Ornithidium already have combinations in those genera, although in some cases their epithets are different than those in Maxillaria. For each species transferred, homotypic synonyms with a different epithet are listed after the basionym when these have been in recent use. Some heterotypic synonyms are given after the abbreviation "syn.".

To further clarify the circumscription of Camaridium, Maxillaria sensu stricto and Ornithidium, we list the names of their constituent species. These lists mostly follow Govaerts et al. (2005) (synonyms not listed) except for cases in which we disagree on synonymization. We have not been able to locate extant type material for many names of Schlechter and other authors published without illustrations; these are not included here, awaiting further research. It is possible that many of these will prove to be synonyms with other better known species.

Most species for which adequate descriptions or type material is available are easy to assign to each genus. However, we prefer to await molecular data to confirm the systematic position of a few species with unusual morphology (e.g., Maxillaria grobyoides Garay \& Dunst., M. muscoides J. T. Atwood, M. poifolia Schltr.).

\section{BRASILIORCHIS}

Brasiliorchis R. Singer, S. Koehler \& Carnevali, Novon 17: 94. 2007.

Type species: Maxillaria picta Hook., = Brasiliorchis picta (Hook.) R. Singer, S. Koehler \& Carnevali. Maxillaria sect. Aggregatae Pfitz. in Engl. \& 
Prantl, Nat. Pflanzenfam. 2(6): 187. 1889, pro parte (excl. type).

Maxillaria sect. Repentes Pfitz. in Engl. \& Prantl, Nat. Pflanzenfam. 2(6): 187. 1889.

Plants of Brasiliorchis have aggregated or distant, sulcate, bifoliate pseudobulbs subtended by non-foliaceous sheaths. Several inflorescences are produced simultaneously from the base of the most recent pseudobulb, and the floral bract is almost always shorter than the pedicel and ovary. The campanulate flowers are food deceptive and do not produce any rewards. The column foot can be short or long. The sepals lack fibers, and in most species they have dark spots, usually more intense on the external surface. The labellum is always markedly three-lobed. The capsules have apical dehiscence ${ }^{2}$.

This well supported clade has been informally known as the "Maxillaria picta alliance"; most species are restricted to Brazil. It was recently described in detail and given generic status by Singer et al. (2007).

Three names need to be commented upon:

Brasiliorchis picta (Hook.) R. Singer, S. Koehler \& Carnevali, Novon 17: 97. 2007.

Maxillaria acutipetala Hook., Bot. Mag. 69: t. 3966. 1842, syn. nov.

Maxillaria acutipetala was described from a plant cultivated at Kew, supposedly collected in Central America by Barclay during the HMS Sulphur expedition. The type specimen and the published painting clearly correspond to the variable Brasiliorchis picta, a taxon reliably documented only from Brazil and northeastern Argentina. The information associated with the plant cultivated at Kew must have been erroneous or confused, and it was most likely not collected by Barclay (the Sulphur explored only the Pacific side of the New World); it was probably collected in Brazil by someone else.

Brasiliorchis barbosae (Loefgr.) R. Singer, S. Koehler \& Carnevali, Novon 17: 96. 2007.

\footnotetext{
${ }^{2}$ Fruits of Maxillariinae always dehisce through six longitudinal seams, but the resulting valves can either remain united both at the base and the tip, or they can separate apically. These two modes of dehiscence are termed "lateral" vs. "apical" (Blanco et al. 2006, Whitten et al. 2007).
}

Basionym: Maxillaria barbosae Loefgr., Arch. Jard. Bot. Rio de Janeiro 2: 60. 1918; 'barbozae'.

Löfgren dedicated the species to João Barbosa Rodrigues but spelled the epithet as "barbozae", which is clearly a typographical error and not an intentional latinization of the Brazilian orchidologist's last name. Both spellings have been used over time, and Singer et al. (2007) used "barbozae" during the generic transfer to Brasiliorchis. However, the epithet should be corrected to "barbosae" under article 60 of the Code (McNeill et al. 2006; also see article 45.2).

Brasiliorchis schunkiana (Campacci \& Kautsky) R. Singer, S. Koehler \& Carnevali, Novon 17: 97. 2007.

Basionym: Maxillaria schunkiana Campacci \& Kautsky, Orquidário 7: 136. 1993; 'schunkeana'.

The adjectival epithet honors Vital Schunk; thus, it must be spelled "schunkiana", not "schunkeana" (Rec. 60C.1, McNeill et al. 2006). Singer et al. (2007) and other authors have used the latter spelling, which is an incorrect orthographical variant.

Species of Brasiliorchis include B. barbosae (Loefgr.) R. Singer, S. Koehler \& Carnevali, $B$. chrysantha (Barb. Rodr.) R. Singer, S. Koehler \& Carnevali, B. consanguinea (Klotzsch) R. Singer, S. Koehler \& Carnevali, B. gracilis (G. Lodd.) R. Singer, S. Koehler \& Carnevali, B. heismanniana (Barb. Rodr.) R. Singer, S. Koehler \& Carnevali, $B$. kautskyi (Pabst) R. Singer, S. Koehler \& Carnevali, B. marginata (Lindl.) R. Singer, S. Koehler \& Carnevali, B. phoenicanthera (Barb. Rodr.) R. Singer, S. Koehler \& Carnevali, B. picta (Hook.) R. Singer, S. Koehler \& Carnevali, B. polyantha (Barb. Rodr.) R. Singer, S. Koehler \& Carnevali, B. porphyrostele (Rchb.f.) R. Singer, S. Koehler \& Carnevali, $B$. schunkiana (Campacci \& Kautsky) R. Singer, S. Koehler \& Carnevali, and B. ubatubana (Hoehne) R. Singer, S. Koehler \& Carnevali (Singer et al. 2007).

\section{Camaridium}

Camaridium Lindl., Bot. Reg. 10: sub t. 844. 1824.

Type species: Camaridium ochroleucum Lindl. Adamanthus Szlach., Richardiana 7: 30. 2007, pro parte (incl. type).

Maxillaria sect. Camaridium (Lindl.) Christenson, Proc. 16 ${ }^{\text {th }}$ World Orchid Conf. 282. 2002. 
Maxillaria sect. Cucullatae Christenson, Proc. $16^{\text {th }}$ World Orchid Conf. 283. 2002.

Maxillaria sect. Pseudomaxillaria (Hoehne) Christenson, Proc. 16 $6^{\text {th }}$ World Orchid Conf. 285. 2002.

Pseudomaxillaria Hoehne, Arq. Bot. Estado Sao Paulo n.s., f.m. 2: 71.1947.

Psittacoglossum LaLlave \& Lex., Nov. Veg. Desc. fasc. 2. (Orch. Opusc.): 29. 1825.

Sauvetrea Szlach., Richardiana 7: 28. 2007, pro parte (excl. type).

Sepalosaccus Schltr., Repert. Spec. Nov. Regni Veg. Beih. 19: 244. 1923.

Species of Camaridium are variable in growth habit; most have pseudobulbs separated by rhizome segments of variable length. Some species are cespitose, and others lack pseudobulbs completely and have monopodial shoots. A few species have dimorphic growth (juvenile sympodial shoots with tightly spaced pseudobulbs, and monopodial mature shoots without pseudobulbs). In almost all species, the floral bract is longer than the pedicel and ovary, and overlaps with the base of the dorsal sepal (this feature is useful in separating Camaridium from Maxillariella and Ornithidium). The column foot can be short or long. The sepals and petals lack fiber bundles and have a sparkling appearance. Most species appear to have deceptive flowers, but some produce nectar. The pendent fruits have apical dehiscence.

As circumscribed here, Camaridium has ca. 80 species distributed throughout the Neotropics, with the highest diversity in Central America. The “Maxillaria neglecta complex" (= Pseudomaxillaria) is nested within Camaridium; the Mesoamerican species were revised by Atwood (1993). The "Maxillaria cucullata complex", which is sister to the rest of Camaridium, is a taxonomically difficult group in dire need of revision.

Camaridium alfaroi (Ames \& C. Schweinf.) M. A. Blanco, comb. nov.

Basionym: Maxillaria alfaroi Ames \& C. Schweinf., Sched. Orch. 10: 83-84. 1930.

Camaridium allenii (L. O. Williams) M. A. Blanco, comb. nov.

Basionym: Maxillaria allenii L. O. Williams, Ann. Missouri Bot. Gard. 27: 282-283, t. 35. 1940.
Camaridium amabile (J. T. Atwood) M. A. Blanco, comb. nov.

Basionym: Maxillaria amabilis J. T. Atwood, Lindleyana 9: 239-241. 1994.

Camaridium ampliflorum (C. Schweinf.) M. A. Blanco, comb. nov.

Basionym: Maxillaria ampliflora C. Schweinf., Bot. Mus. Leafl. 8: 188. 1940. Camaridium grandiflorum Ames, Proc. Biol. Soc. Wash. 34: 149-150. 1921, nom. illeg. [non (Lindl.) Schltr., Repert. Spec. Nov. Regni Veg. Beih. 9: 165. 1921].

Camaridium anceps (Rchb.f.) M. A. Blanco, comb. nov.

Basionym: Ornithidium anceps Rchb.f., Beitr. Orch.K. Centr. Amer. 75-76. 1866. Maxillaria pseudoneglecta J. T. Atwood, Lindleyana 8: 30-31. 1993.

Camaridium atratum (Lex.) M. A. Blanco, comb. nov.

Basionym: Psittacoglossum atratum Lex., Nov. Veg. Descr. 2: 30. 1825. Maxillaria lexarzana Soto Arenas \& F. Chiang, Orquídea (Méx.) n.s., 12: 238239. 1992.

Camaridium aurantiacum (Schltr.) M. A. Blanco, comb. nov.

Basionym: Ornithidium aurantiacum Schltr., Repert. Spec. Nov. Regni Veg. Beih. 19: 241. 1923. Syn.: Maxillaria lankesteri Ames, Sched. Orch. 7: 11-12. 1924. Maxillaria jugata Garay, Orquideología 4: 159. 1969, syn. nov.

Camaridium bomboizense (Dodson) M. A. Blanco, comb. nov.

Basionym: Maxillaria bomboizensis Dodson, Orquideología 19: 59. 1994.

Camaridium brevilabium (Ames \& Correll) M. A. Blanco, comb. nov.

Basionym: Maxillaria brevilabia Ames \& Correll, Bot. Mus. Leafl. 11: 15, fig. 1. 1943. Ornithidium alfaroi Ames \& C. Schweinf., Sched. Orch. 10: 98. 1930. Non Camaridium alfaroi (Ames \& C. Schweinf.) M. A. Blanco.

Camaridium burgeri (J. T. Atwood) M. A. Blanco, comb. nov.

Basionym: Maxillaria burgeri J. T. Atwood, Lindleyana 9: 233-236. 1994. 
Camaridium campanulatum (C. Schweinf.) M. A. Blanco, comb. nov.

Basionym: Maxillaria campanulata C. Schweinf., Bot. Mus. Leafl. 5: 94-95. 1938.

Camaridium cedralense (J. T. Atwood \& Mora-Ret.) M. A. Blanco, comb. nov.

Basionym: Maxillaria cedralensis J. T. Atwood \& Mora-Ret., Selbyana 18: 31-32. 1997.

Camaridium cucullatum (Lindl.) M. A. Blanco, comb. nov.

Basionym: Maxillaria cucullata Lindl., Edwards's Bot. Reg. 26: t. 12. 1840.

Camaridium densum (Lindl.) M. A. Blanco, comb. nov.

Basionym: Maxillaria densa Lindl., Edwards's Bot. Reg. 21: t. 1804. 1835.

Camaridium falcatum (Ames \& Correll) M. A. Blanco, comb. nov.

Basionym: Maxillaria falcata Ames \& Correll, Bot. Mus. Leafl. 11: 15. 1943. Ornithidium costaricense Schltr., Repert. Spec. Nov. Regni Veg. 8: 456. 1910. Non Camaridium costaricense Schltr. (Repert. Spec. Nov. Regni Veg. 3: 249. 1907).

Camaridium fragrans (J. T. Atwood) M. A. Blanco, comb. nov.

Basionym: Maxillaria fragrans J. T. Atwood, Selbyana 22: 131. 2001.

Camaridium gomezianum (J. T. Atwood) M. A. Blanco, comb. nov.

Basionym: Maxillaria gomeziana J. T. Atwood, Lindleyana 11: 202-204. 1996.

Camaridium grisebachianum (Nir \& Dod) M. A. Blanco, comb. nov.

Basionym: Maxillaria grisebachiana Nir \& Dod, Orchid. Antill.: 243. 2000.

Camaridium haberi (J. T. Atwood) M. A. Blanco, comb. nov.

Basionym: Maxillaria haberi J. T. Atwood, Selbyana 16: 245. 1995.

Camaridium hagsaterianum (Soto Arenas) M. A. Blanco, comb. nov.

Basionym: Maxillaria hagsateriana Soto Arenas,
Orquídea (Mexico) n.s., 12: 252. 1992.

Camaridium horichii (Senghas) M. A. Blanco, comb. nov.

Basionym: Maxillaria horichii Senghas, Orchidee (Hamburg) 28: 13. 1977.

Camaridium inauditum (Rchb.f.) M. A. Blanco, comb. nov.

Basionym: Maxillaria inaudita Rchb.f., Beitr. Orch.-K. Centr. Am. 76. 1866.

Camaridium insolitum (Dressler) M. A. Blanco, comb. nov.

Basionym: Maxillaria insolita Dressler, Orquideología 14: 204. 1981.

Camaridium lankesteri (Ames) M. A. Blanco, comb. nov. Basionym: Ornithidium lankesteri Ames, Sched. Orch. 4: 52-53. 1923. Maxillaria quadrata Ames \& Correll, Bot. Mus. Leafl. 6:16. 1943.

Camaridium longicolumna (J. T. Atwood) M. A. Blanco, comb. nov.

Basionym: Maxillaria longicolumna J. T. Atwood, Selbyana 22: 132. 2001.

The epithet longicolumna is a noun in apposition, and therefore retains its feminine gender.

Camaridium lutheri (J. T. Atwood) M. A. Blanco, comb. nov.

Basionym: Maxillaria lutheri J. T. Atwood, Selbyana 19: 257. 1998.

Camaridium meleagris (Lindl.) M. A. Blanco, comb. nov. Basionym: Maxillaria meleagris Lindl., Edwards's Bot. Reg. 30: misc. 3. 1844.

Camaridium micranthum M. A. Blanco, nom. nov. Scaphyglottis parviflora Poepp. \& Endl., Nov. Gen. ac Sp. 1: 58, t. 97. 1835. Maxillaria parviflora (Poepp. \& Endl.) Garay, Bot. Mus. Leafl. 21: 258. 1967. Non Camaridium parviflorum Fawc. (Symb. Antill. 1: 472. 1910).

Camaridium microphyton (Schltr.) M. A. Blanco, comb. nov.

Basionym: Maxillaria microphyton Schltr., Repert. Spec. Nov. Regni Veg. 8: 457. 1910.

Camaridium mombachoense (A. H. Heller ex J. T. Atwood) M. A. Blanco, comb. nov. 
Basionym: Maxillaria mombachoensis A. H. Heller ex J. T. Atwood, Selbyana 5: 302. 1981.

Camaridium monteverdense (J. T. Atwood \& G. Barboza) M. A. Blanco, comb. nov.

Basionym: Maxillaria monteverdensis J. T. Atwood \& G. Barboza, Lindleyana 9: 241-242. 1994.

Camaridium neglectum (Schltr.) M. A. Blanco, comb. nov.

Basionym: Ornithidium neglectum Schltr., Repert. Spec. Nov. Regni Veg. Beih. 19: 242. 1923.

Camaridium obscurum (Linden \& Rchb.f.) M. A. Blanco, comb. nov.

Basionym: Maxillaria obscura Linden \& Rchb.f., Beitr. Orch.-K. Centr. Amer. 31-32, t. 6. 1866.

Camaridium oestlundianum (L. O. Williams) M. A. Blanco, comb. nov.

Basionym: Maxillaria oestlundiana L. O. Williams, Amer. Orchid Soc. Bull. 11: 133. 1942.

Camaridium paleatum (Rchb.f.) M. A. Blanco, comb. nov.

Basionym: Ornithidium paleatum Rchb.f., Linnaea 41: 36.1877.

Camaridium praestans (Rchb.f.) M. A. Blanco, comb. nov.

Basionym: Maxillaria praestans Rchb.f., Gard. Chron., n.s. 23: 566. 1885.

Camaridium pygmaeum M. A. Blanco, nom. nov. Ornithidium wercklei Schltr., Repert. Spec. Nov. Regni Veg. Beih. 19: 60. 1923. Maxillaria wercklei (Schltr.) L. O. Williams, Ann. Missouri Bot. Gard. 27: 284. 1923. Non Camaridium wercklei Schltr. (Repert. Spec. Nov. Regni Veg. Beih. 19: 58-59. 1923).

Camaridium ramonense (Schltr.) M. A. Blanco, comb. nov.

Basionym: Ornithidium ramonense Schltr., Repert. Spec. Nov. Regni Veg. Beih. 19: 243-244. 1923. Maxillaria flava Ames, Hubbard \& C. Schweinf., Bot. Mus. Leafl. 3: 41. 1934.

Camaridium rhombeum (Lindl.) M. A. Blanco, comb. nov.

Basionym: Maxillaria rhombea Lindl., Edwards's Bot. Reg. 26: t. 12. 1840.
Camaridium scalariforme (J. T. Atwood) M. A. Blanco, comb. nov.

Basionym: Maxillaria scalariformis J. T. Atwood, Selbyana 19: 257. 1998.

Camaridium sigmoideum (C. Schweinf.) M. A. Blanco, comb. nov.

Basionym: Ornithidium sigmoideum C. Schweinf., Bot. Mus. Leafl. 4: 121-122. 1937.

Camaridium soconuscanum (Breedlove \& D. Mally) M. A. Blanco, comb. nov.

Basionym: Maxillaria soconuscana Breedlove \& D. Mally, Amer. Orchid Soc. Bull. 58: 1234. 1989.

Camaridium standleyi M. A. Blanco, nom. nov. Maxillaria parvilabia Ames \& C. Schweinf., Sched. Orch. 8: 62-64. 1925, nom. illeg. (non Rolfe, Orchid Rev. 26: 232. 1918).

Camaridium stenophyllum (Schltr.) M. A. Blanco, comb. nov.

Basionym: Ornithidium stenophyllum Schltr., Repert. Spec. Nov. Regni Veg. Beih. 19: 59. 1923. Maxillaria concavilabia Ames \& Correll, Bot. Mus. Leafl. 11: 15, fig. 2. 1953. Non Maxillaria stenophylla Rchb.f. (Bonplandia 2: 17. 1854), nec Maxillaria stenophylla F. Lehm. \& Kraenzl. (Bot. Jahrb. Syst. 26: 481. 1899).

Camaridium strumatum (Endres \& Rchb.f.) M. A. Blanco, comb. nov.

Basionym: Ornithidium strumatum Endres \& Rchb.f., Gard. Chron. 2: 772. 1874.

Camaridium suaveolens (Barringer) M. A. Blanco, comb. nov.

Basionym: Maxillaria suaveolens Barringer, Brittonia 37: 44-46. 1985.

Camaridium synsepalum (J. T. Atwood) M. A. Blanco, comb. nov.

Basionym: Maxillaria synsepala J. T. Atwood, Selbyana 19: 260. 1998.

Camaridium tigrinum (C. Schweinf.) M. A. Blanco, comb. nov.

Basionym: Maxillaria tigrina C. Schweinf., Amer. Orchid Soc. Bull. 37: 409-410. 1968. 
Camaridium tricarinatum (J. T. Atwood) M. A. Blanco, comb. nov.

Basionym: Maxillaria tricarinata J. T. Atwood, Selbyana 19: 260. 1998.

Camaridium tuberculare (J. T. Atwood) M. A. Blanco, comb. nov.

Basionym: Maxillaria tubercularis J. T. Atwood, Lindleyana 9: 229-231. 1994.

Camaridium tutae (J. T. Atwood) M. A. Blanco, comb. nov.

Basionym: Maxillaria tutae J. T. Atwood, Selbyana 19: 262.1998.

Camaridium vaginale (Rchb.f.) M. A. Blanco, comb. nov.

Basionym: Maxillaria vaginalis Rchb.f., Beitr. Orch.-K. Centr. Amer. 77. 1866.

Camaridium valerioi (Ames \& C. Schweinf.) M. A. Blanco, comb. nov.

Basionym: Maxillaria valerioi Ames \& C. Schweinf., Sched. Orch. 10: 96-97. 1930.

Camaridium vittariifolium (L. O. Williams) M. A. Blanco, comb. nov.

Basionym: Maxillaria vittariifolia L. O. Williams, Ceiba 4: 38. 1953.

Other members of Camaridium include C. adolphi Schltr., C. amparoanum Schltr. (Maxillaria serrulata Ames \& Correll), C. biolleyi (Schltr.) Schltr., C. bracteatum (Schltr.) Schltr., C. bradeorum Schltr., C. brenesii Schltr. (M. trilobata Ames \& C. Schweinf.), C. costaricense Schltr. (M. tonduzii (Schltr.) Ames \& Correll), C. ctenostachys (Rchb.f.) Schltr., C. dendrobioides Schltr., C. dichotomum Schltr., C. hoehnei Pabst (M. imbricata Barb. Rodr.), C. imbricatum Schltr. (M. schlechteriana J. T. Atwood), C. latifolium Schltr. (M. planicola C. Schweinf.), C. minus Schltr. [M. minor (Schltr.) L. O. Williams], C. nutantiflorum Schltr. (M. umbratilis L. O. Williams), C. ochroleucum Lindl. (M. camaridii Rchb.f.), and C. pulchrum Schltr.

\section{Christensonella}

Christensonella Szlach., Mytnik, Górniak \& Śmiszek, Polish Bot. J. 51: 57. 2006.

Type species: Maxillaria paulistana Hoehne, =
Christensonella subulata (Lindl.) Szlach., Mytnik, Górniak \& Śmiszek [= Maxillaria subulata Lindl.], syn. nov.

Maxillaria sect. Urceolatae Christenson, Proc. $16^{\text {th }}$ World Orchid Conf. 286-287. 2002.

Species of Christensonella are either epiphytes or lithophytes. Plants of this genus are often very small, compared to other members of Maxillariinae. In most species, the roots show characteristic constrictions. Epiphytic species tend to grow pendent and have distant pseudobulbs, while the lithophytes are erect and more cespitose. The pseudobulbs are often ridged. Each pseudobulb bears from one to four apical leaves, and has several non-foliaceous subtending sheaths. The leaves can be flat and conduplicate to subulate or hemiterete. The flowers are usually yellow to dark red and have a shiny, dry callus; perianth fibers are present. Christensonella uncata and C. squamata have prominent stelidia projecting downward from the apex of the clinandrium, which support a long, tegular stipe. The fusiform fruits have apical dehiscence. In C. nardoides, the endocarpic trichomes are extruded as a sausage-like mass which carries the seeds outside of the fruit (Blanco et al. 2006).

This distinctive group of mostly South American species has been informally known as the "Maxillaria madida alliance" (Pabst \& Dungs 1977). A detailed revision of Christensonella is in progress (S. Koehler, unpublished manuscript). Szlachetko et al. (2006) transferred most members of Maxillaria section Urceolatae to Christensonella, and here we transfer a few more. Whitten et al. (2007) wrongfully cited $M$. nardoides Kraenzl. as the type of Christensonella, but that species is the type of Maxillaria section Urceolatae.

Christensonella cepula (Rchb.f.) S. Koehler, comb. nov. Basionym: Maxillaria cepula Rchb.f., Bonplandia 3: 216. 1855.

Christensonella neowiedii (Rchb.f.) S. Koehler, comb. nov.

Basionym: Maxillaria neowiedii Rchb. f., Linnaea 41:29. 1877.

Christensonella pacholskii (Christenson) S. Koehler, comb. nov. 
Basionym: Maxillaria pacholskii Christenson, Orchid Rev. 111: 288. 2003.

Christensonella squamata (Barb. Rodr.) Carnevali, comb. nov.

Basionym: Maxillaria squamata Barb. Rodr., Gen. Sp. Orchid. 1: 118. 1877.

Other members of Christensonella which should be recognized as discrete species (S. Koehler, unpublished manuscript) include: C. acicularis (Herb. ex Lindl.) Szlach., Mytnik, Górniak \& Śmiszek, $C$. echinophyta (Barb. Rodr.) Szlach., Mytnik, Górniak \& Śmiszek, C. ferdinandiana (Barb. Rodr.) Szlach., Mytnik, Górniak \& Śmiszek, C. nardoides (Kraenzl.) Szlach., Mytnik, Górniak \& Śmiszek, C. pumila (Hook.) Szlach., Mytnik, Górniak \& Śmiszek, C. subulata (Lindl.) Szlach., Mytnik, Górniak \& Śmiszek, C. uncata (Lind1.) Szlach., Mytnik, Górniak \& Śmiszek, and C. vernicosa (Barb. Rodr.) Szlach., Mytnik, Górniak \& Śmiszek. Other names transferred by Szlachetko et al. (2006) are regarded as synonyms (S. Koehler, unpublished manuscript).

\section{Cryptocentrum}

Cryptocentrum Benth., J. Linn. Soc. Bot. 18: 325. 1880.

Type species: Cryptocentrum jamesonii Benth., $=C$. lehmannii (Rchb.f.) Garay [= Aeranthes lehmanii Rchb.f.].

Anthosiphon Schltr., Repert. Spec. Nov. Regni Veg. Beih. 7: 182. 1920.

Cryptocentrum sect. Anthosiphon (Schltr.) Hawkes, Orchid J. 2: 379. 1953.

Cryptocentrum subgen. Caulescentes Senghas, in Schlechter Orchideen, ed. 3, I/B(29): 1798. 1994.

Cryptocentrum subgen. Pseudobulbosa Carnevali, Harvard Pap. Bot. 5: 470. 2001.

Cryptocentrum subgen. Cryptocentrum Benth. sensu Carnevali, Harvard Pap. Bot. 5: 468. 2001.

Pittierella Schltr., Repert. Spec. Nov. Regni Veg. 3(31-32): 80. 1906.

Cryptocentrum is unusual among core Maxillariinae because most species have monopodial (often congested) shoots, long, wiry inflorescences, and greenish, star shaped flowers with a nectariferous spur formed by the bases of the sepals and the labellum. Species in subgenus Caulescentes are even more aberrant, and have dwarf shoots with polystichous phyllotaxis. The capsules have apical dehiscence.

Because of its unusual morphology, Cryptocentrum has been consistently recognized as a separate, well defined genus. Carnevali (2001) provided a synopsis. It is significant that the three subgenera (Caulescentes, Cryptocentrum and Pseudobulbosa; Carnevali, 2001) form strongly supported monophyletic groups (Whitten et al. 2007). Because we now consider Anthosiphon as part of Cryptocentrum, the erection of a new subgenus is necessary:

Cryptocentrum Benth. subgenus Anthosiphon (Schltr.) Carnevali, comb. et stat. nov.

Anthosiphon Schltr., Repert. Spec. Nov. Regni Veg. Beih. 7: 182. 1920.

Cryptocentrum sect. Anthosiphon (Schltr.) Hawkes, Orchid J. 2: 379. 1953.

TYPE: Cryptocentrum roseans (Schltr.) A. D. Hawkes (=Anthosiphon roseans Schltr.)

\section{Cyrtidiorchis}

Cyrtidiorchis Rauschert, Taxon 31: 560. 1982.

Type species (designated by Ortiz, Orquídeas de Colombia ed. 2: 70, 1995): Chrysocycnis rhomboglossa F. Lehm. \& Kraenzl., = Cyrtidiorchis rhomboglossa (F. Lehm. \& Kraenzl.) Rauschert.

Cyrtidium Schltr., Repert. Spec. Nov. Regni Veg. Beih. 27: 178. 1924, nom. illeg. (non Vainio, Acta Soc. Fauna Flora Fenn. 49: 227, 262 1921).

Plants of Cyrtidiorchis have dimorphic growth with sympodial, pseudobulb bearing juvenile shoots, and monopodial, branched adult shoots. Inflorescences are supra-axillary and are only produced by the adult shoots. The flowers have spreading perianth segments that lack fibers, a tomentose, insectiform labellum, and a strongly arched column; they are probably sexually deceptive. The capsules have lateral dehiscence.

Garay (1969) presented a taxonomic revision of Cyrtidiorchis (as Cyrtidium). This small Andean genus (five species) is well characterized and no new combinations are necessary. 


\section{HeterotaXis}

Heterotaxis Lindl., Bot. Reg. 12: t. 1028. 1826.

Type species: Heterotaxis crassifolia Lindl., $=H$. sessilis (Sw.) F. Barros Barros [=Epidendrum sessile $\mathrm{Sw}$.$] .$

Dicrypta Lindl., Gen. Sp. Orchid. Pl. 44. 1830.

Marsupiaria Hoehne, Arq. Bot. Estado Sao Paulo n.s., f.m. 2: 69. 1947.

Maxillaria subgen. Heterotaxis (Lind1.) Brieger, An. Soc. Bot. Brasil 1972: 94. 1972.

Maxillaria sect. Heterotaxis (Lindl.) Brieger, Trab. Congr. Nac. Bot. (Rio de Janeiro) 26: 242-244, 1977.

Maxillaria sect. Iridifolieae Pfitz., Nat. Pflanzenfam. 2(6): 187. 1889.

Pentulops Raf., Fl. Tellur. 4: 42. 1836.

Most species of Heterotaxis have sympodial growth with laterally compressed, oblong, unifoliate pseudobulbs subtended by several foliaceous sheaths. Two species ( $H$. equitans and $H$. valenzuelana) have ensiform leaves and pseudo-monopodial shoots that lack pseudobulbs. In all cases, the shoots are aggregate. The fleshy, yellowish, campanulate flowers have perianth fibers and a very short column foot. The labellum produces a pad of very short, glandular trichomes that likely constitute a reward for pollinators. The capsules have lateral dehiscence.

Ojeda et al. (2005) provided the most recent detailed account of Heterotaxis. They described $H$. fritzii Ojeda \& Carnevali but accidentally provided erroneous information for the type, which is amended here (corrections underlined):

Heterotaxis fritzii Ojeda \& Carnevali, Novon 15: 574-577. 2005.

TYPE: Purchased from Orquídeas del Valle, Cali

[Colombia]. Flowered in cultivation in Gainesville, Florida, U.S.A., 25 Jan 2004, M. W. Whitten 2672 (holotype: FLAS).

Species that belong in Heterotaxis are: H. brasiliensis (Brieger \& Illg) F. Barros, H. discolor (Lodd. ex Lindl.) Ojeda \& Carnevali, H. equitans (Schltr.) Ojeda \& Carnevali, H. fritzii Ojeda \& Carnevali, H. maleolens (Schltr.) Ojeda \& Carnevali, H. microiridifolia (D. E. Benn. \& Christenson) Ojeda \& Carnevali, H. santanae (Carnevali \& I. Ramírez) Ojeda \& Carnevali, H. schul- tesii Ojeda \& G. A. Romero, H. sessilis (Sw.) F. Barros (Maxillaria crassifolia Lindl.), H. superflua (Rchb.f.) F. Barros, H. valenzuelana (A. Rich.) Ojeda \& Carnevali, H. villosa (Barb. Rodr.) F. Barros, and H. violaceopunctata (Rchb.f.) F. Barros (Ojeda et al. 2005).

\section{INTI}

Inti M. A. Blanco, gen. nov.

Type species: Maxillaria chartacifolia Ames \& C. Schweinf., = Inti chartacifolia (Ames \& C. Schweinf.) M. A. Blanco.

Maxillaria sect. Polyphyllae Christenson, Proc. $16^{\text {th }}$ World Orchid Conf. 284-285. 2002.

Plantae cespitosae, epiphyticae, epseudobulbosae, surculis congestis flabellatis. Folia disticha plurima longa angusta. Inflorescentiae axillares in foliis infimis. Flores foetidi, perianthio fibrarum destituto. Fructus capsulares longi dehiscentiis lateralibus.

Plants of Inti are easily recognized by their aggregate, congested shoots devoid of pseudobulbs, with many $(>10)$ distichous, long leaves arranged like a fan. The yellow or maroon flowers lack perianth fibers and have a fetid odor. The column foot is virtually non existent, and the labellum has a pad of glandular trichomes similar to those of Heterotaxis. The capsules are long and narrow, and have lateral dehiscence.

ETyMOLOGY: Named after Inti, the sun god of the Inca culture. The long and narrow leaves radiating from a congested shoot are reminiscent of the rays of a rising sun. Having no botanical tradition, we give this generic name a feminine gender (article 62.3, McNeill et al. 2006).

This small group was treated as the "Maxillaria bicallosa clade" in Whitten et al. (2007).

Inti bicallosa (Rchb.f.) M. A. Blanco, comb. nov.

Basionym: Zygopetalum bicallosum Rchb.f., Otia Bot. Hamb. 1: 9. 1878. Syn.: Ornithidium dolichophyllum Schltr., Repert. Spec. Nov. Regni Veg. Beih. 9: 106. 1921 (= Maxillaria caespitosa C. Schweinf.), syn. nov.

Inti chartacifolia (Ames \& C. Schweinf.) M. A. Blanco, comb. nov.

Basionym: Maxillaria chartacifolia Ames \& C. Schweinf., Sched. Orch. 10: 92. 1930. Syn.: 
Trigonidium equitans Garay, Svensk Bot. Tidskr. 47: 288, fig. 15. 1953, syn. nov.

\section{MAPINGUARI}

Mapinguari Carnevali \& R. Singer, gen. nov.

Type species: Maxillaria longipetiolata Ames \& C. Schweinf., = Mapinguari longipetiolatus (Ames \& C. Schweinf.) Carnevali \& R. Singer.

Plantae plerumque sylvicolae, epiphyticae vel rupicolae, pseudobulbis laevis, semper unifoliatis, aggregatis. Radices laeves, ferrugineae, brunneae vel albae. Flores erecti, fragrantes, rigidi et sine secretiones; pedicello brevissimo rigidoque. Pollinarium cum tegula et viscidio semilunare.

Mapinguari is a small (four species), primarily Guayanan and Amazonian genus. Mapinguari desvauxianus reaches the Brazilian states of São Paulo and Rio de Janeiro. Two of the species are primarily terrestrial in sandy soils or lithophytic on sandstone. The pseudobulbs are aggregate, unifoliate and smooth to slightly sulcate. The conduplicate leaves normally display a well developed petiole. The very short inflorescences are produced from the base of the most recent pseudobulb. The flowers are erect (i.e., the labellum is held in a vertical or near-vertical position), brown or maroon in coloration, have a very short column foot, lack any secretions or trichomes, and have tough perianth fibers. We have not been able to determine the mode of dehiscence of the capsules.

ETYMOLOGY: Named after the Mapinguarí, a legendary and elusive creature of Brazilian-Amazonian mythology, in allusion to the brownish, cryptic flowers. Having no botanical tradition, we treat this generic name as masculine (article 62.3, McNeill et al. 2007).

This small but distinctive group was first recognized by Carnevali \& Ramírez (1989; as the "Maxillaria auyantepuiensis complex") and was treated as the "Maxillaria desvauxiana clade" in Whitten et al. (2007). Full synonymy for each species can be found in Carnevali \& Ramírez-Morillo (2003).

Mapinguari auyantepuiensis (Foldats) Carnevali \& R. Singer, comb. nov.

Basionym: Maxillaria auyantepuiensis Foldats, Bol. Soc. Venez. Ci. Nat. 22: 269. 1961.
Mapinguari desvauxianus (Rchb.f.) Carnevali \& R. Singer, comb. nov.

Basionym: Maxillaria desvauxiana Rchb.f., Bonplandia (Hannover) 3: 67. 1854.

Mapinguari foldatsianus (Carnevali \& I. Ramírez) Carnevali \& R. Singer, comb. nov.

Basionym: Maxillaria foldatsiana Carnevali \& I. Ramírez, Ann. Missouri Bot. Gard. 76: 376. 1989.

Mapinguari longipetiolatus (Ames \& C. Schweinf.) Carnevali \& R. Singer, comb. nov.

Basionym: Maxillaria longipetiolata Ames \& C. Schweinf., Sched. Orch. 8: 61-62. 1925.

\section{MaXillaria}

Maxillaria Ruiz \& Pav., Fl. Peruv. Prodr. 116, t. 25. 1794.

Type species (designated by Brieger \& Hunt, Taxon 18: 601-603. 1969; and by Garay, Harvard Pap. Bot. 11: 51-52. 1997): Maxillaria platypetala Ruiz \& Pav.

Adamanthus Szlach., Richardiana 7: 30. 2007, pro parte (excl. type).

Dendrobium sect. Maxillaria (Ruiz \& Pav.) Pers., Syn. P1. (Persoon) 2: 523. 1807.

Maxillaria sect. Aggregatae Pfitz., Nat. Pflanzenfam. 2(6): 187. 1889, pro parte (incl. type). Maxillaria sect. Amazonicae Christenson, Proc. $16^{\text {th }}$ World Orchid Conf. 282. 2002.

Maxillaria sect. Arachnites Christenson, Proc. $16^{\text {th }}$ World Orchid Conf. 282. 2002.

Maxillaria sect. Axilliflorae Lindl., Gen. Sp. Orchid. P1. 142. 1833, pro parte.

Maxillaria sect. Maxillaria Ruiz \& Pav. sensu Christenson, Proc. $16^{\text {th }}$ World Orchid Conf. 284. 2002.

Maxillaria sect. Multiflorae Christenson, Proc. $16^{\text {th }}$ World Orchid Conf. 284. 2002.

Maxillaria subgen. Aggregatae (Pfitz.) Brieger, Trab. Congr. Nac. Bot. (Rio de Janeiro) 26: 244. 1977.

Menadena Raf., Fl. Tellur. 2: 98. 1836.

Sauvetrea Szlach., Richardiana 7: 28. 2007, pro parte (excl. type).

Plants of this group almost always have pseudobulbs (with the exception of some species in the $M$. 
exaltata alliance) and are often cespitose. The pseudobulbs almost invariably are unifoliate, smooth and laterally compressed, with subtending sheaths that are either foliaceous or not. The abscission layer of the apical leaf is often projected above the pseudobulb in a persistent stalk (phyllopodium). The inflorescences always emerge from the base of the youngest pseudobulbs (from the leaf axils near the top of the stem in the M. exaltata alliance). The floral bract can be shorter or longer than the pedicel and ovary. The flowers have a prominent column foot, and abundant perianth fibers. None of the species produce nectar, but many produce pseudopollen in the form of moniliform, pluricellular trichomes on the labellum surface (a character not seen in any other genera). The capsules have lateral dehiscence.

Almost half of the species traditionally treated in Maxillaria sensu lato will remain in Maxillaria sensu stricto, as here circumscribed. Maxillaria sections Amazonicae, Maxillaria, and Multiflorae form well supported clades (Whitten et al. 2007), but the other sections are polyphyletic. Increased sampling of taxa and gene regions within Maxillaria sensu stricto is needed to support a revised infrageneric classification. Two names included in the analyses of Whitten et al. (2007) need to be commented upon:

Maxillaria candida Lodd. ex Lindl., Edwards's Bot. Reg. 27 (Misc.): 28. 1841.

Syn.: Maxillaria modesta Brade, Orquídea (Rio de Janeiro) 6: 18. 1943, nom. illeg. (non Schltr., Repert. Spec. Nov. Regni Veg. Beih. 28: 93. 1924), Maxillaria modestiflora Pabst, Bradea 2: 319. 1979. syn. nov.

Whitten et al. (2007) reported a specimen of Maxillaria candida Lodd. ex Lindl. (Koehler 0335, ESA) nested in the Mormolyca clade. Upon examination of the type of $M$. candida at Kew, we realized that Koehler 0335 is Mormolyca cf. acutifolia (Lindl.) M. A. Blanco. Whitten et al. (2007) also reported a specimen of Maxillaria modesta Schltr. nested in Maxillaria sensu stricto (Koehler 0351, UEC). This specimen is, however, the true Maxillaria candida, which was originally identified simply as " $M$. modesta". The illegitimate name Maxillaria modesta Brade (non Schltr.) and its later legitimate name M. modestiflora Pabst are previously undetected synonyms of $M$. candida.
Even in this exclusive circumscription, Maxillaria remains by far the largest genus in subtribe Maxillariinae. Species that belong in Maxillaria sensu stricto include: M. acostae Schltr., M. aequiloba Schltr., M. albata Lindl., M. albiflora Ames \& C. Schweinf., M. amazonica Schltr., M. anatomorum Rchb.f., M. angustisegmenta Ames \& C. Schweinf., $M$. angustissima Ames, T. Hubb. \& C. Schweinf., $M$. arachnites Rchb.f., M. arachnitiflora Ames \& C. Schweinf., M. argyrophylla Poepp. \& Endl., M. attenuata Ames \& C. Schweinf., M. atwoodiana Pupulin, M. augustae-victoriae F. Lehm. \& Kraenzl., M. aurorae D. E. Benn. \& Christenson, M. azulensis D. E. Benn. \& Christenson, M. batemanii Poepp. \& Endl., M. bennettii Christenson, M. bocazensis D. E. Benn. \& Christenson, M. bolivarensis C. Schweinf., M. brachybulbon Schltr., M. bradei Schltr. ex Hoehne, M. breviscapa Poepp. \& Endl., M. buchtienii Schltr., M. burtonii D. E. Benn. \& Christenson, M. calantha Schltr., M. candida Lodd. ex Lindl., M. carolii Christenson, $M$. chionantha J. T. Atwood, M. chlorantha Lindl., M. christensonii D. E. Benn., M. colemanii Carnevali \& Fritz, M. colorata Rchb.f., M. confusa Ames \& C. Schweinf., M. connellii Rolfe, M. crocea Poepp. \& Endl., M. cryptobulbon Carnevali \& J. T. Atwood, $M$. curvicolumna M. A. Blanco \& Neubig, M. cuzcoensis C. Schweinf., M. dalessandroi Dodson, $M$. dichroma Rolfe, M. dillonii D. E. Benn. \& Christenson, M. x dunstervillei Carnevali \& I. Ramírez, M. eburnea Lindl., M. ecuadorensis Schltr., M. edwardsii D. E. Benn. \& Christenson, M. elegantula Rolfe, M. embreei Dodson, M. endresii Rchb.f., M. exaltata (Kraenzl.) C. Schweinf., M. fletcheriana Rolfe, M. floribunda Lindl., M. formosa Carnevali \& G. A. Romero, M. fractiflexa Rchb.f., M. frechettei D. E. Benn. \& Christenson, $M$. fucata Rchb.f., M. fuerstenbergiana Schltr., M. galantha J. T. Atwood \& Carnevali, M. gentryi Dodson, $M$. gorbatschowii R. Vásquez, Dodson \& Ibisch, M. grandiflora (Kunth) Lindl., M. grandimentum C. Schweinf., M. grandis Rchb.f., M. granditenuis D. E. Benn. \& Christenson, M. grayi Dodson, M. guadalupensis Cogn., M. hastulata Lindl., M. hennisiana Schltr., $M$. hillsii Dodson, M. hirsutilabia D. E. Benn. \& Christenson, M. huanucoensis D. E. Benn. \& Christenson, M. huebschii Rchb.f., M. irrorata Rchb.f., M. jostii Dodson, M. jucunda F. Lehm. \& Kraenzl., M. kegelii Rchb.f., M. klugii C. Schweinf., M. langlassei 
Schltr., M. leforii D. E. Benn. \& Christenson, $M$. lehmannii Rchb.f., M. lepidota Lindl., M. leucaimata Barb. Rodr., M. lilliputiana D. E. Benn. \& Christenson, M. lindleyana Schltr. (M. crocea Lindl.), M. linearis Ames \& C. Schweinf., M. litensis Dodson, M. longilo$b a$ (Ames \& C. Schweinf.) J. T. Atwood, M. longipes Lindl., M. longissima Lind1., M. loretoensis C. Schweinf., M. lueri Dodson, M. luteoalba Lindl., $M$. macrura Rchb.f., M. margretiae R. Vásquez, M. marmoliana Dodson, M. mejiae Carnevali \& G. A. Romero, M. melina Lindl., M. merana Dodson, $M$. meridensis Lindl., M. microtricha Schltr., M. milenae V. P. Castro \& Chiron, M. molitor Rchb.f., M. monantha Barb. Rodr., M. multiflora Barb. Rodr., M. mungoschraderi R. Vásquez \& Ibisch, M. nanegalensis Rchb.f., M. neophylla Rchb.f., M. niesseniae Christenson, M. nigrescens Lindl., M. nuriensis Carnevali \& I. Ramírez, M. nutans Lindl., $M$. ochroleuca Lodd. ex Lindl., M. pachyneura F. Lehm. \& Kraenzl., M. pannieri Foldats, M. parkeri Hook., M. parvibulbosa C. Schweinf., M. patens Schltr., M. pauciflora Barb. Rodr., M. pentura Lindl., M. perryae Dodson, M. platyloba Schltr., M. platypetala Ruiz \& Pav., M. plicata Schltr., M. porrecta Lindl., M. portillae Christenson, M. powellii Schltr., M. pseudoreichenheimiana Dodson, M. pterocarpa Barb. Rodr., M. pulla Linden \& Rchb.f., M. pyhalae D. E. Benn. \& Christenson, M. quelchii Rolfe, M. ramonensis Schltr., M. reichenheimiana Endres \& Rchb.f., M. ringens Rchb.f., M. rodriguesii Cogn., M. rodrigueziana J. T. Atwood \& Mora-Ret., M. rotundilabia C. Schweinf., M. rubioi Dodson, M. sanderiana Rchb.f. ex Sander, M. setigera Lindl., M. silvana Campacci, M. simacoana Schltr., M. simplicilabia C. Schweinf., M. speciosa Rchb.f., M. spiritu-sanctensis Pabst, M. splendens Poepp. \& Endl., M. striata Rolfe, M. tenuis C. Schweinf., M. thurstoniorum Dodson, M. tiaraensis Carnevali \& G. A. Romero, M. tonsbergii Christenson, M. trilobulata D. E. Benn. \& Christenson, M. triloris E. Morren, M. tristis Schltr., M. tuerosii D. E. Benn. \& Christenson, M. turkeliae Christenson, M. valleculata D. E. Benn. \& Christenson, M. venusta Linden \& Rchb.f., M. whittenii Dodson, M. williamsii Dodson, M. winaywaynaensis D. E. Benn. \& Dodson, $M$. wojii Christenson, M. woytkowskii C. Schweinf., M. xylobiiflora Schltr., M. yanganensis Dodson, and $M$. yauaperyensis Barb. Rodr.

\section{MaXiLlaRiella}

Maxillariella M. A. Blanco \& Carnevali, gen. nov. Type species: Maxillaria diuturna Ames \& C. Schweinf., = Maxillariella diuturna (Ames \& C. Schweinf.) M. A. Blanco \& Carnevali. Adamanthus Szlach., Richardiana 7: 30. 2007, pro parte (excl. type).

Maxillaria sect. Ebulbes Pfitz., Nat. Pflanzenfam. 2(6): 187. 1889

Maxillaria sect. Erectae Pfitz., Nat. Pflanzenfam. 2(6): 187. 1889.

Plantae epiphyticae, pro parte maxima rhizomatibus longis, pseudobulbosae vel epseudoblbosae; pseudobulbi 1-2 foliis apicalibus. Flores solitarii in axillis foliorum vel bractearum. Flores plerumque parvi campanulati. Labella parviloba vel elobulata, callis nitidis. Fructus capsulares dehiscentiis lateralibus.

Species of Maxillariella are variable in terms of growth habit; a few species are subcespitose, but most have pseudobulbs separated by medium to long rhizome segments. The ovoid pseudobulbs are either uni- or bifoliate. Several species with long rhizomes have foliaceous bracts covering the segments between pseudobulbs. Others have pseudobulbs reduced or even absent, and these species show a clear gradient from sympodial to monopodial growth. The most derived members of this genus are the species formerly treated as Maxillaria section Ebulbes (the "Maxillaria graminifolia suballiance", Atwood 2003), which have thin, wiry, monopodial stems completely devoid of pseudobulbs, and narrow, acute leaves. Invariably, only one flower is produced from each leaf or bract axil (e.g., the inflorescences are not fasciculate nor produced sequentially), and the floral bract is shorter than the pedicel and ovary. The column foot is very short, and the labellum is simple or obscurely three-lobed and has a glossy callus. The flowers seem to be food deceptive in most cases. The capsules have lateral dehiscence.

This group was treated as the "Maxillaria variabilis clade" by Whitten et al. (2007). Maxillaria sect. Ebulbes (the "Maxillaria graminifolia suballiance") was revised by Atwood (2003). Camaridium dendrobioides, the type of Adamanthus, is morphologically 
convergent with members of that suballiance, but it is firmly nested in Camaridium (Whitten et al. 2007). The floral bract in $C$. dendrobioides is longer than the pedicel and ovary.

Etymology: The name Maxillariella is a diminutive of Maxillaria, in reference to the often much smaller flowers of Maxillariella.

Maxillariella acervata (Rchb.f.) M. A. Blanco \& Carnevali, comb. nov.

Basionym: Maxillaria acervata Rchb.f., Bonplandia (Hannover) 3: 217. 1855.

Maxillariella alba (Hook.f.) M. A. Blanco \& Carnevali, comb. nov.

Basionym: Dendrobium album Hook.f., Exot. Fl. t. 142. 1825.

Maxillariella anceps (Ames \& C. Schweinf.) M. A. Blanco \& Carnevali, comb. nov.

Basionym: Maxillaria anceps Ames \& C. Schweinf., Sched. Orch. 10: 84. 1930.

Maxillariella appendiculoides (C. Schweinf.) M. A. Blanco \& Carnevali, comb. nov.

Basionym: Maxillaria appendiculoides C. Schweinf., Bot. Mus. Leafl. 4: 119-121. 1937.

Maxillariella arbuscula (Lind1.) M. A. Blanco \& Carnevali, comb. nov.

Basionym: Camaridium arbuscula Lindl., P1. Hartw. 153. 1845.

Maxillariella brevifolia (Lind1.) M. A. Blanco \& Carnevali, comb. nov.

Basionym: Camaridium brevifolium Lindl., Benth. Pl. Hartw. 154. 1845.

Maxillariella caespitifica (Rchb.f.) M. A. Blanco \& Carnevali, comb. nov.

Basionym: Maxillaria caespitifica Rchb.f., Linnaea 41: 73.1877.

Maxillariella cassapensis (Rchb.f.) M. A. Blanco \& Carnevali, comb. nov.

Basionym: Maxillaria cassapensis Rchb.f., Ann. Bot. Syst. 6: 539. 1863.

Maxillariella caucana (Schltr.) M. A. Blanco \& Carnevali, comb. nov.

Basionym: Maxillaria caucana Schltr., Repert. Spec. Nov. Regni Veg. Beih. 7: 167. 1920.
Maxillariella cobanensis (Schltr.) M. A. Blanco \& Carnevali, comb. nov.

Basionym: Maxillaria cobanensis Schltr., Repert. Spec. Nov. Regni Veg. 10: 295. 1912.

Maxillariella costaricensis (Schltr.) M. A. Blanco \& Carnevali, comb. nov.

Basionym: Maxillaria costaricensis Schltr., Rep.

Spec. Nov. Regni Veg. Beih. 19: 232-233. 1923.

Maxillariella curtipes (Hook.) M. A. Blanco \& Carnevali, comb. nov.

Basionym: Maxillaria curtipes Hook., Icon. Pl. 4: t. 384. 1841

Maxillariella densifolia (Poepp. \& Endl.) M. A. Blanco \& Carnevali, comb. nov.

Basionym: Dicrypta densifolia Poepp. \& Endl., Nov. Gen. Sp. Pl. 1: 39. 1836.

Maxillariella diuturna (Ames \& C. Schweinf.) M. A. Blanco \& Carnevali, comb. nov.

Basionym: Maxillaria diuturna Ames \& C. Schweinf., Sched. Orch. 8: 58. 1925.

Maxillariella elatior (Rchb.f.) M. A. Blanco \& Carnevali, comb. nov.

Basionym: Dicrypta elatior Rchb.f., Linnaea 18: 403. 1844.

Maxillariella estradae (Dodson) M. A. Blanco \& Carnevali, comb. nov.

Basionym: Maxillaria estradae Dodson, Icon. Pl. Trop. 1: t. 152. 1980.

Maxillariella funicaulis (C. Schweinf.) M. A. Blanco \& Carnevali, comb. nov.

Basionym: Maxillaria funicaulis C. Schweinf., Bot. Mus. Leafl. 11: 273. 1945.

Maxillariella graminifolia (Kunth) M. A. Blanco \& Carnevali, comb. nov.

Basionym: Isochilus graminifolius Kunth, Nov. Gen. Sp. 1: 340, t. 78. 1816.

Maxillariella guareimensis (Rchb.f) M. A. Blanco \& Carnevali, comb. nov.

Basionym: Maxillaria guareimensis Rchb.f., Bonplandia (Hannover) 2: 16. 1854.

Maxillariella houtteana (Rchb.f.) M. A. Blanco \& Carnevali, comb. nov. 
Basionym: Maxillaria houtteana Rchb.f., Hamb. Gartenz. 14: 212. 1858.

Maxillariella infausta (Rchb.f.) M. A. Blanco \& Carnevali, comb. nov.

Basionym: Maxillaria infausta Rchb.f., Bonplandia (Hannover) 3: 216. 1855.

Maxillariella lawrenceana (Rolfe) M. A. Blanco \& Carnevali, comb. nov.

Basionym: Camaridium lawrenceanum Rolfe, Bull. Misc. Inform. Kew 1894: 185. 1894.

Maxillariella linearifolia (Ames \& C. Schweinf.) M.

A. Blanco \& Carnevali, comb. nov.

Basionym: Maxillaria linearifolia Ames \& C. Schweinf., Sched. Orch. 10: 95-96. 1930.

Maxillariella longibracteata (Lindl.) M. A. Blanco \& Carnevali, comb. nov.

Basionym: Camaridium longibracteatum Lindl., Benth. Pl. Hartw. 154. 1845.

Maxillariella luteorubra (F. Lehm. \& Kraenzl.) M. A. Blanco \& Carnevali, comb. nov.

Basionym: Ornithidium luteorubrum F. Lehm. \& Kraenzl., Bot. Jahrb. Syst. 26: 486. 1899. Maxillaria cuencana Garay, Cand. J. Bot. 34: 257. 1956. Non Camaridium luteo-rubrum Lindl. (Orchid. Linden. 22. 1846).

Maxillariella mexicana (J. T. Atwood) M. A. Blanco \& Carnevali, comb. nov.

Basionym: Maxillaria mexicana J. T. Atwood, Selbyana 24: 35-36. 2003.

Maxillariella microdendron (Schltr.) M. A. Blanco \& Carnevali, comb. nov.

Basionym: Maxillaria microdendron Schltr., Repert. Spec. Nov. Regni Veg. Beih. 8: 94.1921.

Maxillariella nitidula (Rchb.f.) M. A. Blanco \& Carnevali, comb. nov.

Basionym: Maxillaria nitidula Rchb.f., Linnaea 41: 29. 1876.

In Whitten et al. (2007) we stated that this species probably belongs in Camaridium. Upon re-examination of herbarium material, we are now convinced it belongs in Maxillariella.

Maxillariella oreocharis (Schltr.) M. A. Blanco \&
Carnevali, comb. nov.

Basionym: Maxillaria oreocharis Schltr., Repert. Spec. Nov. Regni Veg. Beih. 17: 69. 1922.

Maxillariella pardalina (Garay) M. A. Blanco \& Carnevali, comb. nov.

Basionym: Maxillaria pardalina Garay, Bot. Mus. Leafl. 26: 28. 1978. Maxillaria pantherina Rchb.f. 1855, Bonplandia (Hannover) 3: 239. 1855, nom. illeg. (non Hoffmanns., Verz. Orchid. ed. 3: 71. 1844).

Maxillariella pastensis (Rchb.f.) M. A. Blanco \& Carnevali, comb. nov.

Basionym: Maxillaria pastensis Rchb.f., Bonplandia (Hannover) 3: 239. 1855.

Maxillariella ponerantha (Rchb.f.) M. A. Blanco \& Carnevali, comb. nov.

Basionym: Maxillaria ponerantha Rchb.f., Bonplandia (Hannover) 2: 17. 1854.

Maxillariella procurrens (Lindl.) M. A. Blanco \& Carnevali, comb. nov.

Basionym: Maxillaria procurrens Lindl., Ann. Mag. Nat. Hist. 15: 383. 1845.

Maxillariella prolifera ( $\mathrm{Sw}$.) M. A. Blanco \& Carnevali, comb. nov.

Basionym: Epidendrum proliferum Sw., Prodr.: 124. 1788. Maxillaria swartziana C. D. Adams, Amer. Orchid Soc. Bull. 35: 998. 1966.

Maxillariella purpurata (Lindl.) M. A. Blanco \& Carnevali, comb. nov.

Basionym: Camaridium purpuratum Lindl., Orchid. Linden.: 22. 1846.

Maxillariella robusta (Barb. Rodr.) M. A. Blanco \& Carnevali, comb. nov.

Basionym: Camaridium robustum Barb. Rodr., Gen. Spec. Orchid. 2: 210. 1881. Maxillaria johannis Pabst, Sellowia 10: 165. 1959.

Maxillariella sanguinea (Rolfe) M. A. Blanco \& Carnevali, comb. nov.

Basionym: Maxillaria sanguinea Rolfe, Bull. Misc. Inform. Kew 1895: 8.

Maxillariella spilotantha (Rchb.f.) M. A. Blanco \& Carnevali, comb. nov.

Basionym: Maxillaria spilotantha Rchb.f., Bonplandia (Hannover) 2: 17. 1854. 
Maxillariella stenophylla (Rchb.f. ) M. A. Blanco \& Carnevali, comb. nov.

Basionym: Maxillaria stenophylla Rchb.f., Bonplandia (Hannover) 2: 17. 1854. Non F. C. Lehm. ex Kraenzl. (Bot. Jahrb. Syst. 26: 481. 1899).

Maxillariella stictantha (Schltr.) M. A. Blanco \& Carnevali, comb. nov.

Basionym: Maxillaria stictantha Schltr., Repert. Spec. Nov. Regni Veg. Beih. 8: 97. 1921.

Maxillariella tenuifolia (Lind1.) M. A. Blanco \& Carnevali, comb. nov.

Basionym: Maxillaria tenuifolia Lindl., Edwards's Bot. Reg. 23: sub. t. 1986. 1837.

Maxillariella tuerckheimii (Schltr.) M. A. Blanco \& Carnevali, comb. nov.

Basionym: Camaridium tuerckheimii Schltr., Repert. Spec. Nov. Regni Veg. 10: 296. 1912. Maxillaria nagelii L. O. Williams ex Correll, Lloydia 10: 212. 1947. Non Maxillaria tuerckheimii Schltr. (Repert. Spec. Nov. Regni Veg. 10: 295. 1912).

Maxillariella variabilis (Bateman ex Lindl.) M. A. Blanco \& Carnevali, comb. nov.

Basionym: Maxillaria variabilis Bateman ex Lindl., Edwards's Bot. Reg. 23: sub. t. 1986. 1837.

Maxillariella vinosa (Rolfe) M. A. Blanco \& Carnevali, comb. nov.

Basionym: Camaridium vinosum Rolfe, Bull. Misc. Inform. Kew 1922: 25-26. 1922. Non Camaridium vinosum Schltr. (Repert. Spec. Nov. Regni Veg. Beih. 19: 240. 1923), nec Maxillaria vinosa Senghas (Schlechter Orchideen, ed. 3, I/B(28): 1751. 1993, nom. illeg.).

Maxillariella vulcanica (F. Lehm. \& Kraenzl.) M. A. Blanco \& Carnevali, comb. nov.

Basionym: Maxillaria vulcanica F. Lehm. \& Kraenzl., Bot. Jahrb. Syst. 26: 484. 1899.

Maxillariella xanthorhoda (Schltr.) M. A. Blanco \& Carnevali, comb. nov.

Basionym: Maxillaria xanthorhoda Schltr., Notizbl. Bot. Gart. Berlin-Dahlem 7: 279. 1918.

Maxillariella x yucatanensis (Carnevali \& R. Jiménez) M. A. Blanco \& Carnevali, comb. nov.
Basionym: Maxillaria x yucatanensis Carnevali \& R. Jiménez, Harvard Pap. Bot. 5: 428. 2001.

\section{MORMOLYCA}

Mormolyca Fenzl, Denkschr. Kaiserl. Akad. Wiss., Wien. Math.-Naturwiss. K1. 1: 253. 1850.

Type species: Mormolyca lineolata Fenzl, = M. ringens (Lindl.) Gentil [=Trigonidium ringens Lindl.]. Chrysocycnis Linden \& Rchb.f., Bonplandia (Hannover) 2: 280. 1854.

Cyrtoglottis Schltr., Repert. Spec. Nov. Regni Veg. Beih. 7: 181. 1920.

Maxillaria sect. Rufescens Christenson, Proc. $16^{\text {th }}$ World Orchid Conf. 285-286. 2002.

In this expanded circumscription, Mormolyca can be distinguished by its unifoliate pseudobulbs having a minutely verrucose texture and subtended by non-foliaceous sheaths, the inflorescences arising from the axils of rhizome bracts well behind the most recent pseudobulb, perianth segments that lack fibers and open widely, and a conspicuously clavate, arcuate column. Mormolyca polyphylla (which is sister to the rest of the genus) is atypical because its long, narrow pseudobulbs have up to three apical leaves and two subtending foliaceous sheaths, and the inflorescences are produced from the base of the most recent pseudobulb. The rhizome segments and the floral peduncles can be short or long, depending on the species. The column foot is always very short. The labellum of most species (those formerly treated in Maxillaria section Rufescens) have a pad of short, glandular trichomes on the callus, but the labellum of the other species is tomentose and insectiform. The capsules have apical dehiscence.

Chrysocycnis and Maxillaria section Rufescens are firmly nested within Mormolyca, and thus their constituent species need to be transferred. Mormolyca sensu stricto was revised by Garay \& Wirth (1959), and Chrysocycnis was revised by Sweet (1971). Species of Maxillaria section Rufescens, informally known as the "Maxillaria rufescens complex", constitute a taxonomically difficult group; the Mesoamerican species were revised by Carnevali et al. (2001), but many more are found in South America. There probably are a number of cryptic species, difficult to tell apart as 
herbarium specimens, but distinguishable in life especially by their floral fragrances (Christenson 2002a, 2002b, and personal observation of the authors).

Mormolyca acutifolia (Lindl.) M. A. Blanco, comb. nov. Basionym: Maxillaria acutifolia Lindl., Edwards's Bot. Reg. 25: misc. 92. 1839.

Mormolyca aureoglobula (Christenson) M. A. Blanco, comb. nov.

Basionym: Maxillaria aureoglobula Christenson, Orchids 71: 125-126. 2002.

Mormolyca chacoensis (Dodson) M. A. Blanco, comb. nov.

Basionym: Maxillaria chacoensis Dodson, Icon. Pl. Trop., II, 6: t. 531. 1989.

Mormolyca cleistogama (Brieger \& Illg) M. A. Blanco, comb. nov.

Basionym: Maxillaria cleistogama Brieger \& Illg, Trab. Congr. Nac. Bot. 26: 247. 1977.

Mormolyca dressleriana (Carnevali \& J. T. Atwood) M. A. Blanco, comb. nov.

Basionym: Maxillaria dressleriana Carnevali \& J. T. Atwood, Lindleyana 11: 29-31. 1996.

Mormolyca hedwigiae (Hamer \& Dodson) M. A. Blanco, comb. nov.

Basionym: Maxillaria hedwigiae Hamer \& Dodson, Icon. Pl. Trop. 8: t. 800. 1982.

Mormolyca lehmanii (Rolfe) M. A. Blanco, comb. nov. Basionym: Chrysocycnis lehmanii Rolfe, Bull. Misc. Inform. Kew 1918: 235.

Mormolyca moralesii (Carnevali \& J. T. Atwood) M. A. Blanco, comb. nov.

Basionym: Maxillaria moralesii Carnevali \& J. T. Atwood, Lindleyana 11: 31-32. 1996.

Mormolyca pudica (Carnevali \& Tapia-Muñoz) M. A. Blanco, comb. nov.

Basionym: Maxillaria pudica Carnevali \& TapiaMuñoz, Brittonia 53: 463-465. 2001. Maxillaria rufescens var. minor Fawcett \& Rendle, J. Bot. 48: 108. 1910.

Mormolyca richii (Dodson) M. A. Blanco, comb. nov. Basionym: Maxillaria richii Dodson, Orquideología 19: 81. 1994.
Mormolyca rufescens (Lindl.) M. A. Blanco, comb. nov. Basionym: Maxillaria rufescens Lindl., Edwards's Bot. Reg. 22: t. 1848. 1836.

Mormolyca sanantonioensis (Christenson) M. A. Blanco, comb. nov.

Basionym: Maxillaria sanantonioensis Christenson, Orchids 71: 128. 2002.

Mormolyca schlimii (Linden \& Rchb.f.) M. A. Blanco, comb. nov.

Basionym: Chrysocycnis schlimii Linden \& Rchb.f., Bonplandia (Hannover) 2: 280. 1854.

Mormolyca sotoana (Carnevali \& Gómez-Juárez) M. A. Blanco, comb. nov.

Basionym: Maxillaria sotoana Carnevali \& Gómez-Juárez, Brittonia 53: 461-463.

Mormolyca suarezorum (Dodson) M. A. Blanco, comb. nov.

Basionym: Maxillaria suarezorum Dodson, Icon. Pl. Trop., II, 6: t. 547. 1989.

Mormolyca tenuibulba (Christenson) M. A. Blanco, comb. nov.

Basionym: Maxillaria tenuibulba Christenson, Orchid Rev. 109: 41. 2001.

The other members of the genus constitute the paraphyletic Mormolyca sensu stricto: M. aurorae D. E. Benn. \& Christenson, M. gracilipes (Schltr.) Garay \& M. Wirth, M. peruviana C. Schweinf., M. polyphylla Garay \& M. Wirth, $M$. ringens (Lindl.) Gentil, and $M$. schweinfurthiana Garay \& M. Wirth. The obscure name M. galeata (Scheidw.) Garay \& M. Wirth is not a true Mormolyca, but probably represents a species in the Camaridium cucullatum alliance.

\section{Nitidobulbon}

Nitidobulbon Ojeda, Carnevali \& G. A. Romero, ined.

Species of this group have oblong, shiny, smooth pseudobulbs with several (4-6) subtending foliaceous sheaths, and one or two apical leaves. The flowers are campanulate and the rigid perianth segments have abundant fibers. The column foot is very short, and the labellum is frequently reflexed at the tip. The labellar callus is ligulate and secretes an abundant, 
resinous substance, but lacks glandular trichomes. The capsules have lateral dehiscence.

This small group was referred to as the "Maxillaria nasuta clade" in Whitten et al. (2007), and will be elevated to generic rank by Ojeda et al. (in press). It comprises the following three species currently in Maxillaria: M. cymbidioides Dodson, J. T. Atwood \& Carnevali, M. nasuta Rchb.f., and M. proboscidea Rchb.f.

\section{ORNITHIDIUM}

Ornithidium Salisb. ex R. Br., Hort. Kew. ed. 2, 5: 210. 1813.

Type species: Epidendrum coccineum Jacq., = Ornithidium coccineum (Jacq.) Salisb. ex R. Br.

Adamanthus Szlach., Richardiana 7: 30. 2007, pro parte (excl. type).

Laricorchis Szlach., Richardiana 7: 27. 2007.

Maxillaria sect. Ornithidium (Salisb. ex R. Br.) Christenson, Richardiana 2: 52. 2002.

Maxillaria sect. Reflexae Christenson, Proc. $16^{\text {th }}$ World Orchid Conf. 285. 2002.

Maxillaria sect. Siagonanthus (Poepp. \& Endl.) Christenson, Proc. 16 ${ }^{\text {th }}$ World Orchid Conf. 286. 2002.

Neo-urbania Fawc. \& Rendle, J. Bot. 47: 125. 1909.

Siagonanthus Poepp. \& Endl., Nov. Gen. Sp. Pl. (Poeppig \& Endlicher) 1: 40. 1836.

Species of Ornithidium can be either sympodial (cespitose to long-rhizomatous) or monopodial; a few species have dimorphic growth (sympodial juvenile shoots and monopodial adult shoots). The stems and leaves of most species have an olive green coloration, which is persistent upon drying. When present, the ovoid pseudobulbs have a shiny, minutely cracked texture reminiscent of old varnish. The thick roots have a characteristic orangish coloration. Inflorescences are usually fascicled, and the pedicel and ovary invariably are much longer than the floral bract. The flowers are usually small, fleshy, campanulate or more often subglobose, and often produce nectar, and the perianth lacks fibers. Many species have yellow, orange, or red flowers. Capsules have apical dehiscence.
Ornithidium adendrobium (Rchb.f.) M. A. Blanco \& Ojeda, comb. nov.

Basionym: Ponera adendrobium Rchb.f., Flora 48: 278. 1964.

Ornithidium affine (Poepp. \& Endl.) M. A. Blanco \& Ojeda, comb. nov.

Basionym: Scaphyglottis affinis Poepp. \& Endl., Nov. Gen. Sp. P1. 1: 59, t. 99A. 1836.

Ornithidium cachacoense (J. T. Atwood) M. A. Blanco \& Ojeda, comb. nov.

Basionym: Maxillaria cachacoensis J. T. Atwood, Selbyana 24: 30-31. 2003.

Ornithidium canarense (J. T. Atwood) M. A. Blanco \& Ojeda, comb. nov.

Basionym: Maxillaria canarensis J. T. Atwood, Selbyana 24: 31-32. 2003.

Ornithidium condorense (J. T. Atwood) M. A. Blanco \& Ojeda, comb. nov.

Basionym: Maxillaria condorensis J. T. Atwood, Selbyana 24: 32-33. 2003.

Ornithidium fimbriatilobum (Carnevali \& G. A. Romero) M. A. Blanco \& Ojeda, comb. nov.

Basionym: Maxillaria fimbriatiloba Carnevali \& G.

A. Romero, Orchids Venezuela, ed. 2, 3: 11381139. 2000.

Ornithidium gualaquizense (Dodson) M. A. Blanco \& Ojeda, comb. nov.

Basionym: Maxillaria gualaquizensis Dodson, Orquideología 19: 69. 1994.

Ornithidium haemathodes (Ruiz \& Pav.) M. A. Blanco \& Ojeda, comb. nov.

Basionym: Fernandezia haemathodes Ruiz \& Pav., Syst. Veg. Fl. Peruv. Chil. 1: 240. 1798.

Ornithidium lasallei (Foldats) M. A. Blanco \& Ojeda, comb. nov.

Basionym: Maxillaria lasallei Foldats, Contr. Ocas. Mus. Hist. Nat. Colegio de la Salle 3: 2. 1961.

Ornithidium machinazense (D. E. Benn. \& Christenson) M. A. Blanco, comb. nov.

Basionym: Maxillaria machinazensis D. E. Benn. \& Christenson, Lindleyana 13: 71. 1998.

Ornithidium maldonadoense (J. T. Atwood) M. A. Blanco \& Ojeda, comb. nov. 
Basionym: Maxillaria maldonadoensis J. T. Atwood, Selbyana 24: 33-35. 2003.

Ornithidium minutiflorum (D. E. Benn. \& Christenson) M. A. Blanco \& Ojeda, comb. nov.

Basionym: Maxillaria minutiflora D. E. Benn. \& Christenson, Icon. Orchid. Peruv.: t. 700. 2001.

Ornithidium nicaraguense (Hamer \& Garay) M. A. Blanco \& Ojeda, comb. nov.

Basionym: Neo-urbania nicaraguensis Hamer \& Garay, Icon. Pl. Trop. 13: t. 1238. 1985.

Ornithidium oxapampense (J. T. Atwood) M. A. Blanco \& Ojeda, comb. nov.

Basionym: Maxillaria oxapampensis J. T. Atwood, Selbyana 24: 36-37. 2003.

Ornithidium patellum (J. T. Atwood) M. A. Blanco \& Ojeda, comb. nov.

Basionym: Maxillaria patella J. T. Atwood, Selbyana 24: 37-39. 2003.

Ornithidium patulum (C. Schweinf.) M. A. Blanco \& Ojeda, comb. nov.

Basionym: Maxillaria patula C. Schweinf., Fieldiana, Bot. 28: 197. 1951.

Ornithidium pseudonubigenum (J. T. Atwood) M. A. Blanco \& Ojeda, comb. nov.

Basionym: Maxillaria pseudonubigena J. T. Atwood, Selbyana 24: 39-41. 2003.

Ornithidium pustulosum (J. T. Atwood) M. A. Blanco \& Ojeda, comb. nov.

Basionym: Maxillaria pustulosa J. T. Atwood, Selbyana 24: 41-43. 2003.

Ornithidium rauhii (D. E. Benn. \& Christenson) M. A. Blanco \& Ojeda, comb. nov.

Basionym: Maxillaria rauhii D. E. Benn. \& Christenson, J. Orchideenfr. 12: 34. 2005.

Ornithidium repens (L. O. Williams) M. A. Blanco \& Ojeda, comb. nov.

Basionym: Maxillaria repens L. O. Williams, Amer. Orchid Soc. Bull. 10: 273. 1942.

Ornithidium rigidum (Barb. Rodr.) M. A. Blanco \& Ojeda, comb. nov.

Basionym: Maxillaria rigida Barb. Rodr., Gen. Spec. Orchid. 2: 206. 1881.
Ornithidium scandens (D. E. Benn. \& Christenson) M. A. Blanco \& Ojeda, comb. nov.

Basionym: Maxillaria scandens D. E. Benn. \& Christenson, Icon. Orchid. Peruv.: t. 707. 2001.

Ornithidium scullianum (J. T. Atwood) M. A. Blanco \& Ojeda, comb. nov.

Basionym: Maxillaria sculliana J. T. Atwood, Selbyana 24: 1-3. 2003.

Ornithidium sillarense (Dodson \& Vásquez) M. A. Blanco \& Ojeda, comb. nov.

Basionym: Maxillaria sillarensis Dodson \& Vásquez, Icon. Pl. Trop., II, 3: pl. 261. 1989.

Ornithidium simplex (J. T. Atwood) M. A. Blanco \& Ojeda, comb. nov.

Basionym: Maxillaria simplex J. T. Atwood, Selbyana 24: 43. 2003.

Other members of Ornithidium include O. aggregatum Rchb.f., O. aureum Poepp. \& Endl., O. breve Schltr. (Maxillaria palmensis Dodson), O. chrysocycnoides Schltr., O. coccineum (Jacq.) Salisb. ex R. Br., O. conduplicatum Ames \& C. Schweinf., $O$. croceorubens Rchb.f., O. distichum Lindl., O. fulgens Rchb.f., O. giganteum Lindl., O. hystrionicum Rchb.f., O. jamesonii Rchb.f., O. mapiriense Kraenzl., O. miniatum Lindl., O. multicaule (Poepp. \& Endl.) Rchb.f., O. niveum Lindl., O. nubigenum Rchb.f., O. pendens (Pabst) Senghas, O. pendulum (Poepp. \& Endl.) Cogn., O. pittieri Ames, O. purpureolabium (D. E. Benn. \& Christenson) Senghas, O. quitense Rchb.f., O. ruberrimum (Lindl.) Rchb.f., O. sanaense (D. E. Benn. \& Christenson) Senghas, $O$. semiscabrum Lindl., O. serrulatum Lindl. ( $M$. alticola C. Schweinf.), O. sophronitis Rchb.f., $O$. squarrosum Schltr., and O. tonsoniae (Soto Arenas) Senghas.

\section{Pityphyllum}

Pityphyllum Schltr., Repert. Spec. Nov. Regni Veg. Beih. 7: 162. 1920.

Type species (designated by Sweet, 1972: 205): Pityphyllum antioquiense Schltr.

Plants of Pityphyllum are minute, pendulous epiphytes, with long rhizome segments between the tiny pseudobulbs. The pseudobulbs are completely covered and fused 
to a subtending sheath (tunica), and can have from one to 20 apical leaves, depending on the species. There is a pair of ligular projections at the apex of each leaf sheath, next to the abscission layer of the blade. The diminutive flowers are borne from the axils of rhizome bracts between the pseudobulbs. The column foot is virtually non existent, and the capsules have apical dehiscence.

This small and distinctive Andean genus was revised by Sweet (1972) and recently expanded by Whitten et al. (2006) to accommodate P. huancabambae (Kraenzl.) Whitten and P. saragurense (Dodson) Whitten, previously placed in Maxillaria. The other species are $P$. amesianum Schltr., $P$. antioquiense Schltr., P. hirtzii Dodson, P. laricinum (Kraenzl.) Schltr., and $P$. pinoides Sweet.

\section{RHETINANTHA}

Rhetinantha M. A. Blanco, gen. nov.

Type species: Maxillaria acuminata Lindl., = Rhetinantha acuminata (Lindl.) M. A. Blanco.

Sauvetrea Szlach., Richardiana 7: 28. 2007, pro parte (excl. type).

Plantae epiphyticae, rhizomatibus longis et pseudobulbis 1-4 foliis apicalibus vel plantae pendulae foliis equitantibus. Flores plerumque virides, nunquam late expansi, partibus perianthii rigidis acuminatis. Labella elobulata, secretionibus resinosis vel ceraceis. Fructus capsulares dehiscentiis lateralibus.

Plants of Rhetinantha are sub-cespitose to long rhizomatous; the pseudobulbs are often ridged, and have two to four apical leaves, and usually one or two subtending foliaceous sheaths. The inflorescences often arise from rhizome bracts a few shoots behind the most recent pseudobulb. The flowers are campanulate, with rigid, acuminate perianth parts with strong fibers, and a very short column foot. The labellum secretes a sticky, resinous substance in most species. The margins of the clinandrium are conspicuously ciliate, and the pollinarium stipe is long with involute margins. A few species have a pair of stelidia projected downward from the apex of the clinandrium. The capsules have lateral dehiscence. Rhetinantha witsenioides is highly modified and has pendent, monopodial shoots with ensiform, glaucous leaves.

Eтymology: From the Greek words "rhetinos" (resin) and "anthos" (flower), in reference to the flowers of most species, which secrete resin on the labellum and occasionally on the petals.

This well defined group was treated as the "Maxillaria acuminata clade" by Whitten et al. (2007).

Rhetinantha aciantha (Rchb.f.) M. A. Blanco, comb. nov. Basionym: Maxillaria aciantha Rchb.f., Bot. Zeitung (Berlin) 10: 858. 1852.

Rhetinantha acuminata (Lindl.) M. A. Blanco, comb. nov.

Basionym: Maxillaria acuminata Lindl., Pl. Hartw. 155. 1845. Sauvetrea acuminata (Lindl.) Szlach., Richardiana 7: 29. 2006.

Rhetinantha cerifera (Barb. Rodr.) M. A. Blanco, comb. nov.

Basionym: Maxillaria cerifera Barb. Rodr. Gen. Spec. Orchid. 1: 118. 1877.

Rhetinantha divaricata (Barb. Rodr.) M. A. Blanco, comb. nov.

Basionym: Ornithidium divaricatum Barb. Rodr., Gen. Spec. Orchid. 2: 209. 1881.

Rhetinantha encyclioides (J. T. Atwood \& Dodson) M. A. Blanco, comb. nov.

Basionym: Maxillaria encyclioides J. T. Atwood \& Dodson, Orquideología 20: 268. 1997.

Rhetinantha friedrichsthalii (Rchb.f) M. A. Blanco, comb. nov.

Basionym: Maxillaria friedrichsthalii Rchb.f., Bot. Zeitung (Berlin) 10: 858. 1852.

Rhetinantha mariaisabeliae (J. T. Atwood) M. A. Blanco, comb. nov.

Basionym: Maxillaria mariaisabeliae J. T. Atwood, Selbyana 7: 250. 1984.

Rhetinantha monacensis (Kraenzl.) M. A. Blanco, comb. nov.

Basionym: Maxillaria monacensis Kraenzl., Repert. Spec. Nov. Regni Veg. 24: 49. 1927.

Rhetinantha neilii (Dodson) M. A. Blanco, comb. nov.

Basionym: Maxillaria neilii Dodson, Orquideología 19: 79.1994. 
Rhetinantha notylioglossa (Rchb.f.) M. A. Blanco, comb. nov.

Basionym: Maxillaria notylioglossa Rchb.f., Bonplandia (Hannover) 2: 16. 1854.

Rhetinantha ophiodens (J. T. Atwood) M. A. Blanco, comb. nov.

Basionym: Maxillaria ophiodens J. T. Atwood, Selbyana 7: 248. 1984.

Rhetinantha pastorellii (D. E. Benn. \& Christenson) M. A. Blanco, comb. nov.

Basionym: Maxillaria pastorellii D. E. Benn. \& Christenson, Lindleyana 13: 74. 1998. Maxillaria unguiculata D. E. Benn. \& Christenson, Brittonia 47: 197. 1995, nom. illeg. (non Schltr., Repert. Spec. Nov. Regni Veg. Beih. 7: 173. 1920).

Rhetinantha schistostele (Schltr.) M. A. Blanco, comb. nov.

Basionym: Maxillaria schistostele Schltr., Repert. Spec. Nov. Regni Veg. Beih. 19: 303-304. 1923.

Rhetinantha scorpioidea (Kraenzl.) M. A. Blanco, comb. nov.

Basionym: Maxillaria scorpioidea Kraenzl., Svensk. Vet. Akad. Handl. 46: 71. 1911.

Rhetinantha witsenioides (Schltr.) M. A. Blanco, comb. nov.

Basionym: Maxillaria witsenioides Schltr., Repert. Spec. Nov. Regni Veg. Beih. 7: 175. 1920.

\section{SAUVETREA}

Sauvetrea Szlach., Richardiana 7: 28. 2007.

Type species: Maxillaria alpestris Lindl., = Sauvetrea alpestris (Lind1.) Szlach.

Maxillaria sect. Trigonae Christenson, Proc. $16^{\text {th }}$ World Orchid Conf. 286. 2002.

Plants of Sauvetrea are cespitose to moderately longrhizomatous. The unifoliate pseudobulbs are frequently ancipitous and are subtendend by a pair of non-foliaceous bracts. The inflorescences arise from the base of the newly emerging pseudobulbs and have strongly ancipitous bracts. The column foot is short. The flowers have spreading perianth segments without strong fibers. Both the ovaries and the mature fruits are strongly trigonous. The labellum has a ligulate, sulcate callus, and the midlobe is much longer than the lateral lobes. There are no secretions produced by the flowers. The capsules have lateral dehiscence.

Sauvetrea was polyphyletic as originally circumscribed by Szlachetko \& Śmiszek (2007). We already indicated the species that do not belong in this clade (Whitten et al. 2007), and here we transfer some that do.

Sauvetrea bomboizensis (Dodson) M. A. Blanco, comb. nov.

Basionym: Maxillaria bomboizensis Dodson, Orquideología 19(3): 59-61. 1994.

Sauvetrea chicana (Dodson) M. A. Blanco, comb. nov.

Basionym: Maxillaria chicana Dodson, Orquideología 19(3): 61. 1994.

Sauvetrea cornuta (C. Schweinf.) M. A. Blanco, comb. nov.

Basionym: Maxillaria cornuta C. Schweinf., Bot. Mus. Leafl. 11: 265. 1945.

Sauvetrea laevilabris (Lind1.) M. A. Blanco, comb. nov.

Basionym: Maxillaria laevilabris Lind1., P1. Hartw.: 155. 1845. Syn.: Maxillaria piestopus Schltr., Repert. Spec. Nov. Regni Veg. Beih. 19: 302. 1923, syn. nov.; Maxillaria koehleri Schltr., Repert. Spec. Nov. Regni Veg. Beih. 9: 103. 1921, syn. nov.

Sauvetrea machupicchuensis (Christenson) M. A. Blanco, comb. nov.

Basionym: Maxillaria machupicchuensis Christenson, Orchids 71: 718-719. 2002.

Sauvetrea napoensis (Dodson) M. A. Blanco, comb. nov. Basionym: Maxillaria napoensis Dodson, Icon. Pl. Trop., II, 6: t. 540. 1989.

Sauvetrea sessilis (Lind1.) M. A. Blanco, comb. nov. Basionym: Maxillaria sessilis Lindl., Pl. Hartw. 155. 1843.

Sauvetrea trigona subsp. amaroensis (D. E. Benn. \& Christenson) M. A. Blanco, comb. nov.

Basionym: Maxillaria trigona subsp. amaroensis D. E. Benn. \& Christenson, Icon. Orchid. Peruv.: t. 708. 2001.

Other species in the genus include $S$. alpestris 
(Lindl.) Szlach., S. peruviana (C. Schweinf.) Szlach., S. trigona (C. Schweinf.) Szlach., S. unicarinata (C. Schweinf.) Szlach., and S. xantholeuca (Schltr.) Szlach. However, Sauvetrea needs a thorough taxonomic revision. The strange Maxillaria grobyoides Garay \& Dunst. may belong in Sauvetrea, but we prefer to wait until we have molecular data to confirm or reject this hypothesis.

\section{Trigonidium}

Trigonidium Lindl., Bot. Reg. 23: t. 1923. 1837. Type species: Trigonidium obtusum Lindl.

Plants of Trigonidium are either cespitose or longrhizomatous. The ridged pseudobulbs have one to four apical leaves and are subtended by non-foliaceous bracts. The inflorescences are erect, often long and wiry, and emerge from the most recent pseudobulbs. The flowers are erect and campanulate and lack a column foot. The sepals form a cup around the smaller petals and lip and are reflexed at the middle. The petals have characteristic shiny thickenings at the apex. The capsules have lateral dehiscence.

Trigonidium has been consistently recognized as a genus since its creation. No nomenclatural transfers are needed. A synoptical treatment of the genus is in progress (M. A. Blanco, unpublished manuscript).

AcKNOWLEDGEMENTS. Eric A. Christenson (BRIT) provided general discussions on taxonomy and data about the publication of his sections of Maxillaria. Gustavo Romero (AMES), Cassio van den Berg (HUEFS) and two anonmous reviewers provided suggestions for improving the manuscript. Funding was provided by the U.S. National Science Foundation (grant No. DEB-0234064) to N.H.W. and W.M.W., a FAPESP postdoctoral grant (01/08958-1) to R.B.S., a M.Sc. grant from CONACYT to I.O., KLARF fellowships from the Royal Botanic Garden, Kew, to M.A.B. (to study type material in European herbaria) and S.K. (a Prance Fellowship to study systematics of Christensonella), and a Furniss Foundation Ph.D. Fellowship from the American Orchid Society to M.A.B.

\section{Literature Cited}

Atwood, J. T. 1993. A revision of the Maxillaria neglecta complex (Orchidaceae) in Mesoamerica. Lindleyana 8: 25-31.

Atwood, J. T. 2003. Review of the Maxillaria graminifolia (Kunth) Rchb.f. (Orchidaceae) suballiance. Selbyana 24:
144-164.

Backlund, A., \& K. Bremer. 1998. To be or not to be principles of classification and monotypic plant families. Taxon 47: 391-400.

Blanco, M. A., W. M. Whitten, N. H. Williams, \& S. Koehler. 2006. Capillitial extrusion from fruits of Maxillaria nardoides (Orchidaceae: Maxillariinae). Orchids 75: 677-683.

Carnevali, G. 2001. A synoptical view of the classification of Cryptocentrum (Orchidaceae), new taxa, and a key to the genus. Harvard Pap. Bot. 5: 467-486.

Carnevali, G., \& I. Ramírez. 1989. New or noteworthy orchids for the Venezuelan flora. VII. Additions in Maxillaria from the Venezuelan Guayana. Ann. Missouri Bot. Gard. 76: 374-380.

Carnevali, G., \& I. M. Ramírez-Morillo. 2003. Maxillaria. In: G. Carnevali, I.M. Ramírez-Morillo \& G. A. Romero-González (eds.). Orchidaceae. Fl. Venez. Guayana 7: 426.454.

Carnevali, G., J. L. T. Muñoz, \& M. Gómez-Juarez. 2001. A synopsis of the Maxillaria rufescens complex in Mexico, Central America, and the Greater Antilles. Brittonia 53: 454-465.

Chase, M. W., J. V. Freudenstein, K. M. Cameron, \& R. L. Barrett. 2003. DNA data and Orchidaceae systematics: a new phylogenetic classification. In: K. W. Dixon, S. P. Kell, R. L. Barrett, \& P. J. Cribb (eds.). Orchid Conservation. Natural History Publications, Kota Kinabalu, Sabah, Malaysia, 69-89.

Christenson, E. A. 2002a. Maxillaria, an overview. In: J. Clark, W. Elliott, G. Tingley, \& J. Biro (eds.). Proceedings of the $16^{\text {th }}$ World Orchid Conference, Vancouver, 1999. Vancouver Orchid Society, Vancouver, British Columbia, Canada. 279-290.

Christenson, E. A. 2002b. Vue d'ensemble du genre Maxillaria. Richardiana 2: 41-65.

Cribb, P. J., R. Holttum, J. Stewart, G. Seidenfaden, \& E. A. Schelpe. 1985. A note on automatic transfers. Taxon 34: 122-135.

Dathe, S., \& H. Dietrich. 2006. Comparative molecular and morphological studies in selected Maxillariinae orchids. Willdenowia 36: 89-102.

Dressler, R. L. 1993. Phylogeny and classification of the orchid family. Dioscorides Press, Portland, Oregon, USA.

Entwisle, T. J., \& P. H. Weston. 2005. Majority rules, when systematists disagree. Austral. Syst. Bot. 18: 1-6.

Garay, L. A. 1969. Sinopsis del género Cyrtidium Schltr. Orquideología 4: 3-13.

Garay, L. A., \& M. Wirth. 1959. On the genera Mormolyca Fenzl and Cyrtoglottis Schltr. Canad. J. Bot. 37: 479-490.

Govaerts, R., M. A. Campacci, D. Holland Baptista, P. 
Cribb, A. George, K. Kreuz, \& J. Wood. 2005. World Checklist of Orchidaceae. The Board of Trustees of the Royal Botanic Gardens, Kew. http://www.kew.org/wcsp/monocots/, accessed 10 January 2007.

McNeill, J., F. R. Barrie, H. M. Burdet, V. Demoulin, D. L. Hawksworth, K. Marhold, D. H. Nicholson, J. Prado, P. C. Silva, J. E. Skog, W. J. Wiersema, \& N. J. Turland. 2006. International Code of Botanical Nomenclature (Vienna Code). Reg. Veg. 146.

Monro, A. K. 2006. Revision of species-rich genera: A phylogenetic framework for the strategic revision of Pilea (Urticaceae) based on cpDNA, nrDNA, and morphology. Amer. J. Bot. 93: 426-441.

Ojeda, I., G. Carnevali, \& G. A. Romero. 2005. New species and combinations in Heterotaxis Lindley (Orchidaceae: Maxillariinae). Novon 15: 572-582.

Ojeda, I., G. Carnevali Fernández-Concha, \& G. A. Romero. In press. Nitidobulbon, a new genus of Maxillariinae (Orchidaceae). Novon.

Pabst, G. F. J., \& F. Dungs. 1977. Orchidaceae brasilienses. Band 2. Brücke-Verlag Kurt Schmersow, Hildesheim, Germany.

Parnell, J. A. N., L. A. Craven, \& E. Biffin. 2007. Matters of scale: dealing with one of the largest genera of angiosperms. In: T. R. Hodkinson \& J. A. N. Parnell (eds.), Reconstructing the tree of life - Taxonomy and systematics of species rich taxa. CRC Press, Boca Raton, Florida, USA. 251-273.

Pfeil, B. E., \& M. D. Crisp. 2005. What to do with
Hibiscus? A proposed nomenclatural resolution for a large and well known genus of Malvaceae and comments on paraphyly. Austral. Syst. Bot. 18: 49-60.

Ruiz, H., \& J. Pavón. 1794. Prodromus Flora Peruvianae et Chilensis 116. t. 25.

Ruiz, H., \& J. Pavón. 1798. Maxillaria, Systema Vegetabilium Florae Peruvianae et Chilensis 1: 219-226. Gabrielis de Sancha, Madrid, Spain.

Singer, R. B., S. Koehler, \& G. Carnevali. 2007. Brasiliorchis: a new genus for the Maxillaria picta alliance (Orchidaceae: Maxillariinae). Novon 17: 91-99.

Sweet, H. R. 1971. Orquídeas Andinas poco conocidas V. Chrysocycnis Linden \& Rchb.f.. Orquideología 6: 3-10.

Sweet, H. R. 1972. Orquídeas Andinas poco conocidas VI. Pityphyllum. Orquideología 7: 203-214.

Szlachetko, D. L., \& M. Śmiszek. 2007. Noveaux genres dans le complexe Maxillaria (Orchidaceae). Richardiana 7: 26-32.

Szlachetko, D. L., J. Mytnik-Ejsmont, M. Górniak, \& M. Śmiszek. 2006. Genera et species orchidalium. 15. Maxillarieae. Polish Bot. J. 51: 57-59.

Whitten, M. W., M. A. Blanco, \& N. H. Williams. 2006. Recircumscription of Pityphyllum (Orchidaceae: Maxillariinae). Orchids 75: 452-456.

Whitten, W. M., M. A. Blanco, N. H. Williams, S. Koehler, G. Carnevali, R. B. Singer, L. Endara, \& K. M. Neubig. 2007. Molecular phylogenetics of Maxillaria and related genera (Orchidaceae: Cymbidieae) based on combined molecular data sets. Amer. J. Bot. 94: 18601889. 
LANKESTERIANA 